\title{
Using High-Resolution Simulations to Quantify Errors in Radar Estimates of Tornado Intensity
}

\author{
NATHAN A. DAHL \\ School of Meteorology, University of Oklahoma, Norman, Oklahoma \\ DAVID S. NOLAN \\ Rosenstiel School of Marine and Atmospheric Science, University of Miami, Miami, Florida
}

(Manuscript received 6 November 2017, in final form 9 March 2018)

\begin{abstract}
Observation experiments are performed on a set of high-resolution large-eddy simulations of translating tornado-like vortices. Near-surface Doppler wind measurements are taken by emulating a mobile radar positioned from 1 to $10 \mathrm{~km}$ south of each vortex track and conducting single-level scans every $2 \mathrm{~s}$. The departure of each observed gust (wind measurement averaged over two successive scans) from the corresponding true maximum 3-s gust at $10 \mathrm{~m}$ AGL ("S10-3s") is partitioned into error sources associated with resolution volume size, wind direction relative to the radar beam, beam elevation, and temporal sampling. The distributions of each error type are diagrammed as functions of range, observed wind speed, and predicted deviation between the wind direction and the radar beam. The results indicate that the deviation between the wind direction and the radar beam is the predominant source of error in these rapid scan scenarios, although range is also a substantial factor. The median total error is $\sim 10 \%$ for small deviation at close range, but it approximately doubles if the range is increased from 1 to $10 \mathrm{~km}$; a more pronounced increase in both the median value and the variance of the total error is seen as the deviation becomes large. Because of this, the underestimate of the global maximum S10-3s approaches $30-40 \mathrm{~m} \mathrm{~s}^{-1}$ at a longer range, although the global maximum of the time-averaged observed wind speed gives a reasonable approximation of the time-mean maximum S10-3s in many cases. Because of simplifying assumptions and the limited number of cases examined, these results are intended as a baseline for further research.
\end{abstract}

\section{Introduction}

The advent of Doppler radar has dramatically improved tornado warning reliability and lead time. Simmons and Sutter (2005) report that the percentage of tornadoes that prompted warnings in the contiguous United States rose from $35 \%$ to $60 \%$ and the mean tornado warning lead time nearly doubled after the installation of the Weather Surveillance Radar-1988 Doppler (WSR-88D; Crum and Alberty 1993) network. Their regression analysis also indicated that expected fatalities and injuries decreased over the same period by $45 \%$ and $40 \%$, respectively. Among other factors, Brotzge and Erickson (2009) attribute such improvements to the use of Doppler radar data in real-time operations (see also Bieringer and Ray 1996) as well as increased conceptual understanding of tornadic storms

Corresponding author: Nathan A. Dahl,dahl_nathan@ou.edu through field projects employing mobile Doppler radars (e.g., VORTEX-95; Rasmussen et al. 1994).

In addition to detecting the presence of a tornado, Doppler radar data can be used to estimate tornado intensity. For example, the maximum winds within the vortex may be simply approximated from observed velocity extrema, or the observed wind field may be used as a basis for deducing the structure of the vortex though methods such as the ground-based velocity track display (GBVTD) technique (Lee et al. 1999). With the recent development of dual-polarization capabilities, products such as differential reflectivity and copolar correlation coefficient are also being used in conjunction with wind measurements to identify and characterize lofted debris as an indication of damage severity (Bodine et al. 2013; Cheong et al. 2017).

Historically, tornado intensity ratings on the Fujita scale (Fujita 1971), and later the enhanced Fujita (EF) scale (Wind Science and Engineering Center 2006), have 
been based on observed damage, which is then used as a proxy estimate for near-surface wind speed. However, the quality of such estimates is questionable. Twisdale (1982) notes the substantial occurrence of rating errors [e.g., due to uncertainties regarding structural integrity and nonwind effects such as debris impacts; Reynolds (1971); Doswell and Burgess (1988); Marshall (2002); Doswell et al. (2009); Edwards 2003; Edwards et al. (2013)], as well as "random encounter" errors due to the large number of tornadoes that occur in areas with few structures (Schaefer and Galway 1982; Doswell and Burgess 1988). Because mobile Doppler measurements can provide a more comprehensive picture of winds within the tornado (thereby avoiding errors due to unreliable and/or sparse proxy indicators), there have been recent efforts to include radar measurements in the rating process, although their inclusion remains controversial; for example, the radar-based preliminary EF4 rating for the May 2013 Bennington, Kansas, tornado was later downgraded because of "the current directive which states that one cannot rate tornado intensity by nondamage data sources" (NCDC 2013, p. 189).

In considering this topic, it must be noted that tornado intensity estimates based on radar observations have limitations of their own, which are summarized by Snyder and Bluestein (2014). On a basic level, each radar observation is a weighted average of the radarrelative motion of a finite number of scatterers within a resolution volume and thus may be subject to statistical uncertainties as well as substantial bias in the case of a tornado [e.g., from centrifuging; see also Dowell et al. (2005)]. Even if those error sources are ignored, general difficulties remain: 1) lateral beam spreading leads to larger resolution volumes at longer ranges, impairing the detection of smaller-scale structures (e.g., subvortices); 2) the altitude of the center of the beam is typically above the $10 \mathrm{~m}$ AGL level specified for EF scale winds, vertical beam spreading leads to the resolution volume including samples over layer tens or even hundreds of meters deep (even for mobile radar datasets), and recent observations of tornadic surface layers (Kosiba and Wurman 2013; Wurman et al. 2013; Snyder and Bluestein 2014) emphasize that the relationship between the $10-\mathrm{m}$ wind speed and the wind speed at other altitudes is poorly understood; and 3) perhaps most problematic of all, Doppler radars are only capable of observing the component of the wind parallel to the radar beam, so any strong wind lateral to the beam is missed.

It is clearly desirable to quantify each of these error sources. However, using real data to do so requires collocated, contemporaneous wind observations of sufficient quality to verify the radar measurements [e.g., in situ wind measurements within the vortex as in Wurman et al. (2013)] and the hostile, unpredictable nature of tornadoes severely limits opportunities to obtain such measurements. Therefore, as was done for in situ observations in Dahl et al. (2017), we seek such quantification by creating synthetic observations from high-resolution simulations of tornado-like vortices. Section 2 describes the method of generating the vortices, obtaining simulated radar observations, and evaluating various error sources. Section 3 describes the results, while section 4 summarizes the implications and suggests possible extensions of this work.

\section{Experimental method}

\section{a. Generating tornado-like vortices}

In this study, we revisit the set of vortex simulations used by Dahl et al. (2017). The basic details of those simulations are summarized here for convenience: the vortices were generated with Cloud Model 1 (Bryan and Fritsch 2002) using a "Fiedler chamber" approach (Fiedler 1994; Nolan 2005; Rotunno 2013) in which a dry, isentropic fluid is circulated within a closed chamber translating to the east at a speed of $10 \mathrm{~m} \mathrm{~s}^{-1}$ by a constant vertical forcing function $F(x, y, z)$ defined within a "bubble" around the center axis. Fluid originating in the far field, with angular momentum defined by a prescribed background rotation rate $\Omega$, is drawn toward the center axis at low levels, producing an end-wall vortex dynamically and structurally similar to an actual tornado.

To keep the results insensitive to the lateral boundaries of the chamber, a large domain was used $(40 \mathrm{~km} \times$ $40 \mathrm{~km} \times 15 \mathrm{~km}$ ), with lateral and vertical grid stretching employed to afford very high grid resolution $(5 \mathrm{~m} \times$ $5 \mathrm{~m} \times 2.5 \mathrm{~m}$ ) in the vicinity of the vortex, and the flow in the upper part of the domain was damped to eliminate disturbances exiting the central updraft before they could recirculate into the low-level inflow. To reduce computational cost, the vortices were first spun up to nearly steady state on an axisymmetric grid. The result provided the initial conditions for the full 3D simulations, which were run for a further $1800 \mathrm{~s}$ ( $1500 \mathrm{~s}$ to allow the vortex to adjust from the initial axisymmetric steady state and $300 \mathrm{~s}$ to obtain output for analysis).

A semislip lower boundary is specified, and subgrid turbulence is parameterized using a 1.5-order TKE scheme. To better justify a "large-eddy simulation" treatment of the surface layer, we employed an "eddy injection" technique (Bryan et al. 2017) in which resolved eddies were generated separately based on the conditions in the far field and then introduced into the chamber at a radius of $2 \mathrm{~km}$ from the vortex. As shown in Bryan et al. (2017), this method markedly reduces the relative impact 


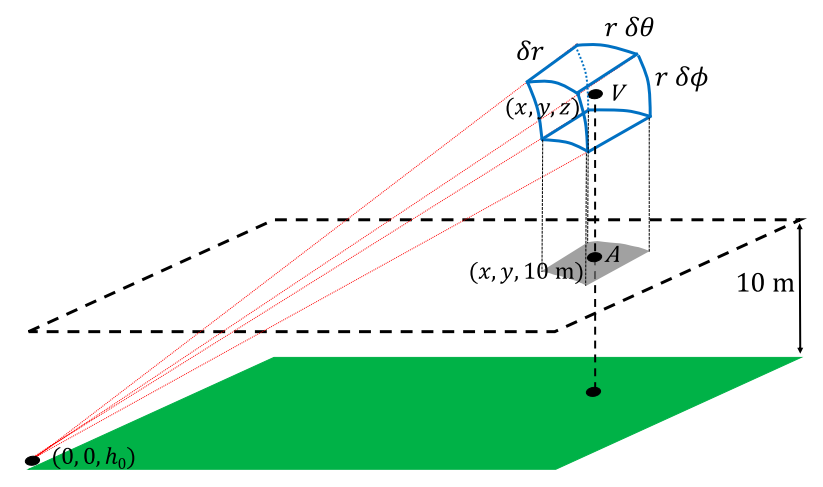

FIG. 1. Example position of verification region $A$ (gray) in relation to resolution volume $V$ (blue) for a given Doppler observation; $h_{0}$ denotes the operating height of the radar.

of subgrid (parameterized) turbulence on the flow within the surface layer, although it is not eliminated; Nolan et al. (2017) show nontrivial contributions to the surface layer momentum budget by subgrid turbulence, although this is generally confined below $10 \mathrm{~m}$ AGL for the grid spacing used in the present study.

(a)

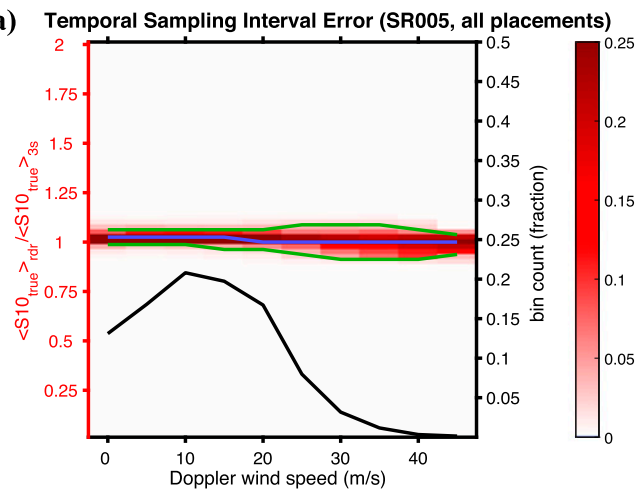

(c) Temporal Sampling Interval Error (SR02, all placements)

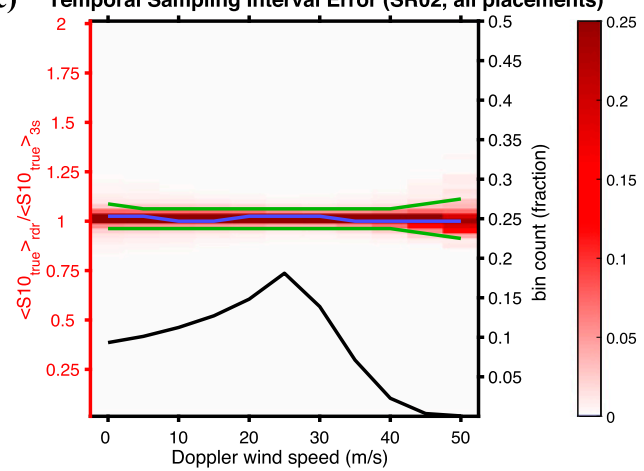

With all other factors held constant, the size and structure of the simulated vortex are largely governed by a swirl ratio:

$$
S_{r}=\frac{\Omega L_{r}}{W},
$$

where $L_{r}=3000 \mathrm{~m}$ is the radius of the forcing function bubble and

$$
W=\sqrt{2 \int_{z=0}^{z=15 \mathrm{~km}} F\left(0,0, z^{\prime}\right) d z^{\prime}}
$$

is the "thermodynamic speed limit" evaluated at the center axis. A larger swirl ratio is characterized by a broader vortex with a greater propensity to break down into multiple subvortices. Based on the results of Dahl et al. (2017), we use simulations with $W=40 \mathrm{~m} \mathrm{~s}^{-1}$ and $S_{r}=0.005$ ("SR005" or "low swirl"), $S_{r}=0.01$ ("SR01" or "medium swirl"), or $S_{r}=0.02$ ("SR02" or "high swirl") to examine a range of vortex types at moderateto-violent intensity. For a complete description of the (b)

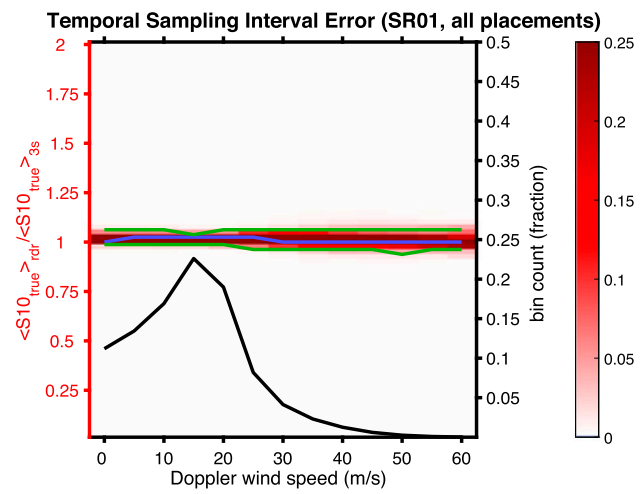

(d)

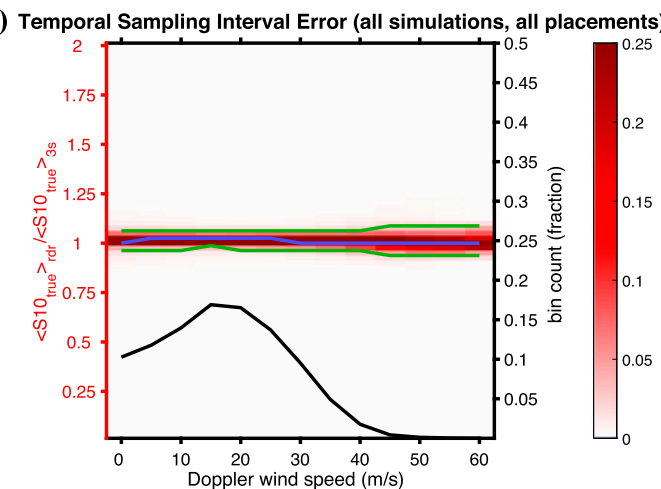

FIG. 2. Bivariate frequency diagrams of temporal sampling error as a function of observed Doppler wind speed, aggregated over all radar placements, for simulation (a) SR005, (b) simulation SR01, (c) simulation SR02, and (d) all simulations. Median values for each wind speed bin are plotted in blue, 5th and 95th percentiles are plotted in green, and the fraction of the total count contained in each bin is plotted in black. 

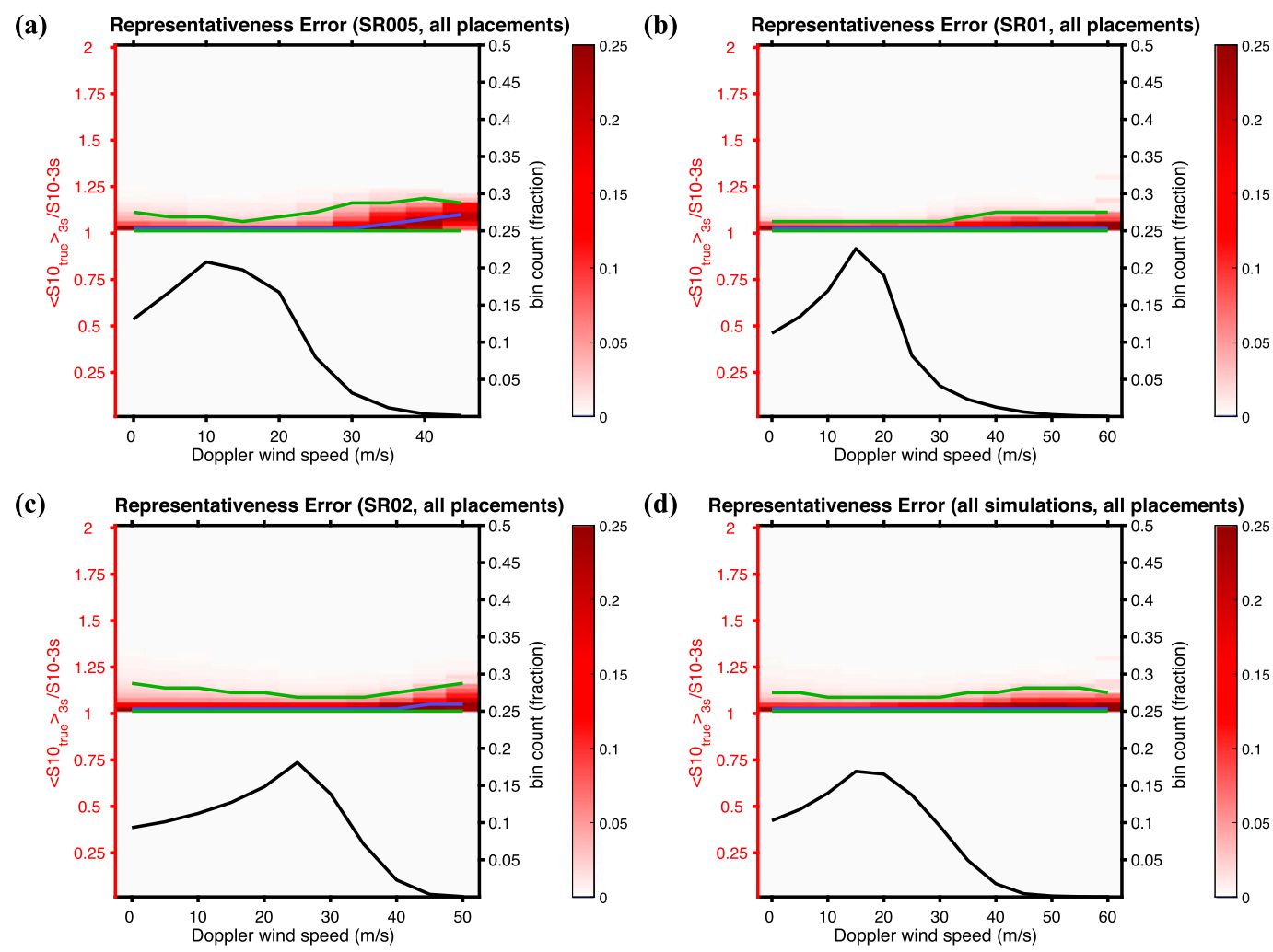

FIG. 3. As in Fig. 2, but for representativeness error.

modeling framework, the reader is referred to Rotunno et al. (2016), Bryan et al. (2017), and Nolan et al. (2017).

\section{b. Simulating radar wind observations}

The vortex simulations are dry and debris free, and while we were able to save the $10 \mathrm{~m}$ AGL winds at 0.1-s intervals for verification purposes, the full 3D state is only available at 1-s intervals over a 300-s period because of cost limitations. Therefore, we employ some simplifying assumptions when simulating the radar observations in order to examine an "optimal" scenario. First, we assume that each radar scan is instantaneous and occurs at the output time, thereby neglecting errors in vortex representation due to the structural changes that occur over the course of a realworld radar scan. In theory, one could seek to account for these errors by using an advection correction technique like the one described in Shapiro et al. (2015) to adjust the model state to the time corresponding to each azimuth in a continuous radar sweep. However, such techniques generally rely on the "frozen turbulence" hypothesis of Taylor (1938), which is of questionable validity within tornadoes (particularly for intense subvortices developing and decaying at time scales on the order of the radar sampling interval), and evaluation of their efficacy for tornadic wind fields is outside the scope of this paper.
To mitigate the disparity between this assumption and real-world radar measurements as much as possible, we choose to emulate the rapid scan, X-band, polarimetric mobile radar (RaXPol; Pazmany et al. 2013) in these experiments. Operating at its maximum scan rate, $\mathrm{RaXPol}$ requires $2 \mathrm{~s}$ to obtain a single $360^{\circ}$ sweep. This scan time is among the shortest available; the Mobile Weather Radar, 2005 X-band, Phased Array (MWR05XP; Bluestein et al. 2010) is also capable of performing 2-s sweeps, but the RaXPol has a smaller beamwidth and is therefore more suited for tornado-scale observations. A full RaXPol volume scan requires $\sim 20$ s, which is substantially longer than the time required by the rapid scan Doppler on Wheels (RSDOW; Wurman and Randall 2001). However, since we are only concerned here with wind measurements very close to the ground, a single low-elevation sweep is sufficient for this work.

For a given resolution volume scanned by the radar, the observed radial wind speed $U_{\text {obs }}$ is the reflectivityweighted average of the radial speeds of the scatterers in the volume. The lower boundary is considered to be transparent to the radar beam and we assume that the model domain contains a uniform distribution of identical, isotropic scatterers that perfectly follow the wind. Thus, we assume that the radar observes only the true 
(a)

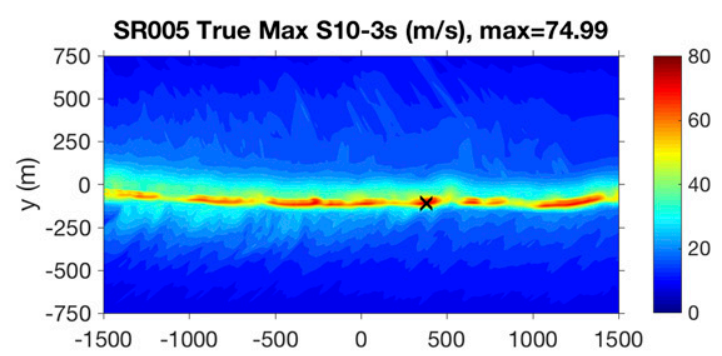

(b) SR005 Max Radar 3s Wind (m/s) at 1 km Range, max $=59.23$

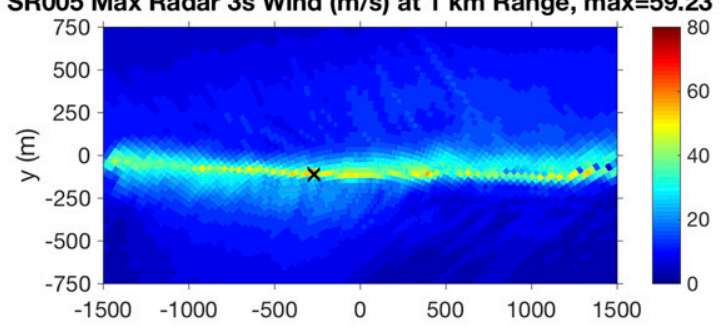

(c) SR005 Max Radar 3s Wind (m/s) at $5 \mathrm{~km}$ Range, $\max =52.22$

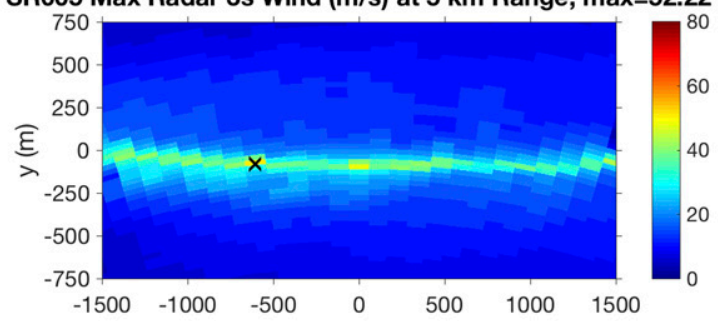

(d) SR005 Max Radar 3s Wind (m/s) at $10 \mathrm{~km}$ Range, max $=36.55$

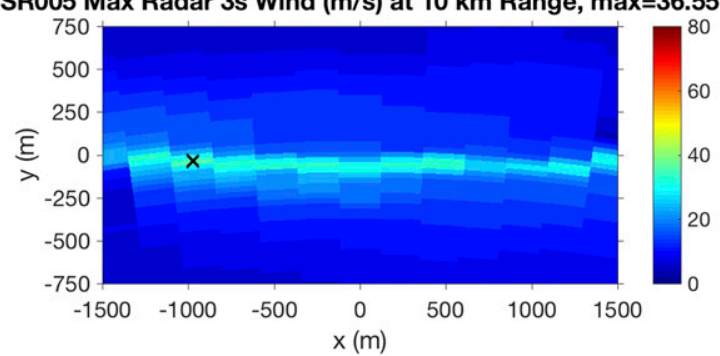

FIG. 4. (a) Swaths of true local maximum 10-m, 3-s gust, and local maximum radar-estimated gust from placements (b) $1 \mathrm{~km}$ south of the track, (c) $5 \mathrm{~km}$ south of the track, and (d) $10 \mathrm{~km}$ south of the track for simulation SR005. The $x$ coordinates are relative to the radar position, while the $y$ coordinates are relative to the center of the model grid.

atmospheric motion at each model grid point and any deviant scatterer motions (due to centrifuging, etc.), effects of nonuniform scatterer distribution (e.g., motion in one region of the volume receiving greater weight than other regions because of more concentrated and/or reflective scatterers), or interactions with the surface (e.g., clutter) are neglected, even though these factors can produce nontrivial errors in real-world radar observations of tornadic winds (Nolan 2013; Snyder and Bluestein 2014). Under these assumptions, we employ a simple weighting method based on the range and the power distribution of the radar beam (similar to the one described in Potvin et al. 2009):

$$
U_{\mathrm{obs}}=\frac{\sum_{i=1}^{N} B_{i} R_{i} U_{i}}{\sum_{i=1}^{N} B_{i} R_{i}},
$$

where $N$ is the number of model grid points contained in the resolution volume, $U_{i}=\mathbf{U}_{i} \cdot \mathbf{r}_{i}$ is the true radial wind at the $i$ th grid point, and $\mathbf{r}=\left[\left(x_{i}-x_{r}\right) \hat{\mathbf{i}}+\left(y_{i}-y_{r}\right) \hat{\mathbf{j}}+\left(z_{i}-z_{r}\right) \hat{\mathbf{k}}\right]$ is the radar-relative position vector. The term $R_{i}$ is the range weight, given by

$$
R_{i}=\left\{\begin{array}{ll}
1, & \left|\Delta r_{i}\right|<0.3 d \\
\min \left(\frac{0.5 d-\left|\Delta r_{i}\right|}{0.2 d}, 0\right), & \left|\Delta r_{i}\right| \geq 0.3 d
\end{array},\right.
$$

where $d$ is the range resolution of the radar [set here to $30 \mathrm{~m}$ to match the value for RaXPol given in Snyder and Bluestein (2014)] and $\Delta r_{i}$ is the difference between $\left|\mathbf{r}_{i}\right|$ and the distance from the radar to the center of the resolution volume. The term $B_{i}$ is the beam weight for the $i$ th model grid point, given by

$$
B_{i}=\exp \left\{-8 \ln 2\left[\left(\frac{\theta_{h i}}{\theta_{B h}}\right)^{2}+\left(\frac{\theta_{v i}}{\theta_{B v}}\right)^{2}\right]\right\},
$$

where $\theta_{h i}$ and $\theta_{v i}$ are the differences in radar-relative azimuth and zenith angles, respectively, between $\mathbf{r}_{i}$ and the center of the beam; and $\theta_{B h}$ and $\theta_{B v}$ are the horizontal and vertical half-power beamwidths, respectively. To emulate RaXPol, we set $\theta_{B v}=1.0^{\circ}$ and $\theta_{B h}=1.4^{\circ}$ to account for the lateral "smearing" produced by the high scan rate as noted in Wakimoto et al. (2015); Pazmany et al. (2013) describe a "strobe technique" to counter the smearing effect, but employing it would increase the minimum measureable range to $1.8 \mathrm{~km}$. Since this would eliminate or substantially reduce observations in the vicinity of the vortex for many of our experiments, we ignore it here to maintain internal consistency. [Note also that this weight pattern neglects any impact by sidelobes, which can also be a significant source of contamination; e.g., see Snyder and Bluestein (2014) and Doviak and Zrnić (1993, p. 105).]

In all cases, the vortex motion is essentially eastward at a speed of $10 \mathrm{~m} \mathrm{~s}^{-1}$ (following the motion of the forcing bubble, which is centered at the model grid origin), and therefore we approximate a priori the vortex track over the 300-s output period as centered at $y=0$ and extending from $x=0$ to $x=3 \mathrm{~km}$. To simulate the observations, we place radars directly south of the midpoint of the track 
(a)

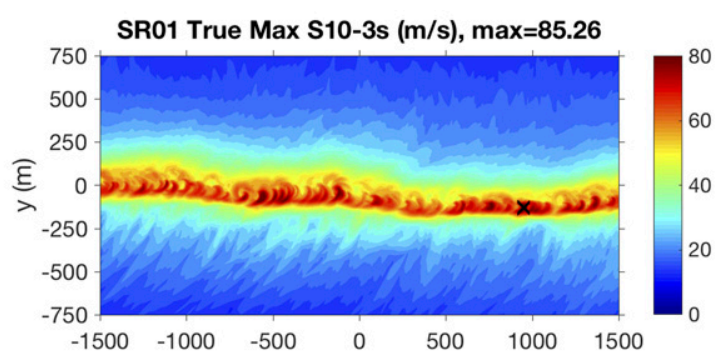

(b) SR01 Max Radar 3s Wind ( $\mathrm{m} / \mathrm{s}$ ) at $1 \mathrm{~km}$ Range, $\max =78.66$

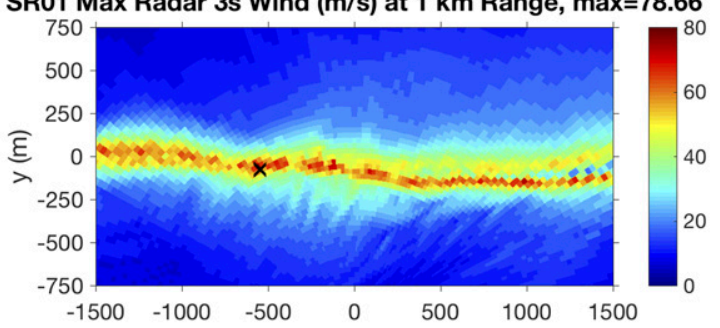

(c) SR01 Max Radar $3 \mathrm{~s}$ Wind $(\mathrm{m} / \mathrm{s})$ at $5 \mathrm{~km}$ Range, $\max =53.77$

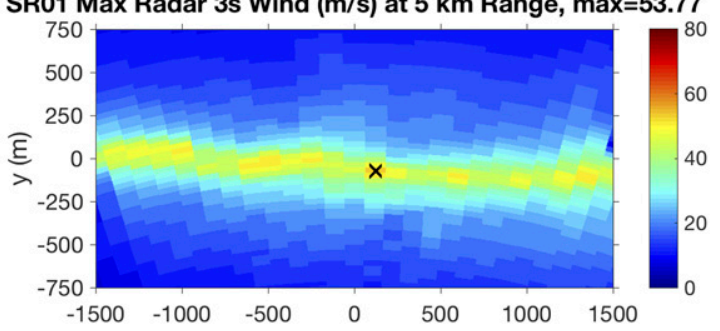

(d) SR01 Max Radar 3s Wind (m/s) at $10 \mathrm{~km}$ Range, $\max =45.59$

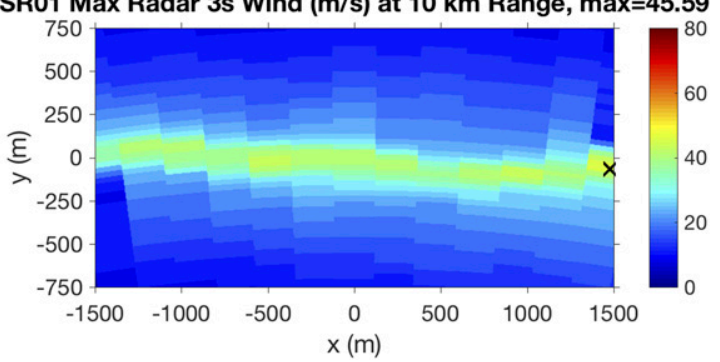

FIG. 5. As in Fig. 4, but for simulation SR01.

(i.e., at $x=1.5 \mathrm{~km}$ ) at distances ranging from 1 to $10 \mathrm{~km}$. Radar beam center height is commonly estimated using the 4/3 Earth's radius model (Doviak and Zrnić 1993). For a small horizontal distance $r$ from the radar and a small beam elevation angle $\phi$, this model reduces to

$$
h(r) \approx h_{0}+r \tan \phi+\frac{r^{2}}{2 k_{e} a \cos \phi},
$$

where $r$ is the horizontal distance to the radar, $h_{0}$ is the operating height of the radar (set here to $2 \mathrm{~m}$ ), $k_{e}=4 / 3$, and $a=6371 \mathrm{~km}$ is Earth's radius. The third term on the right-hand side of (6) accounts for the effects of Earth's curvature and beam refraction. However, at these distances $(r \sim 1-10 \mathrm{~km})$, the deflection due to those effects (a)

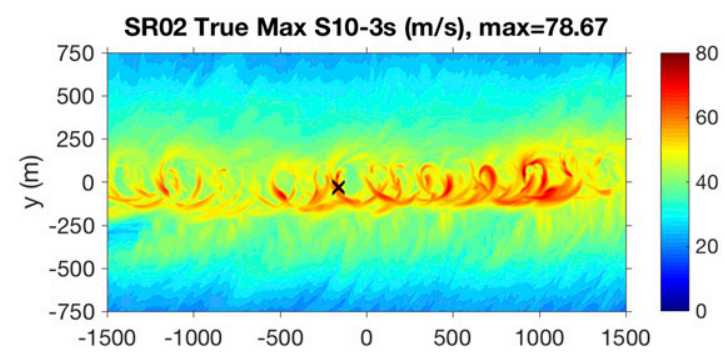

(b) SR02 Max Radar 3s Wind ( $\mathrm{m} / \mathrm{s})$ at $1 \mathrm{~km}$ Range, $\max =63.30$

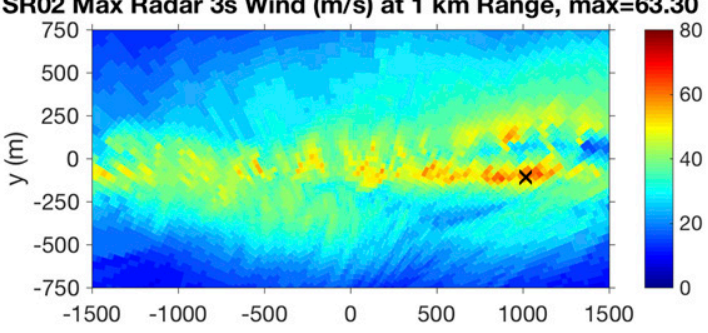

(c) SR02 Max Radar 3s Wind (m/s) at $5 \mathrm{~km}$ Range, $\max =55.05$

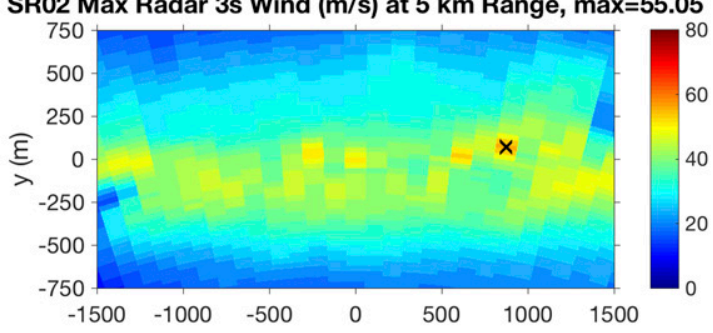

(d) SR02 Max Radar $3 \mathrm{~s}$ Wind $(\mathrm{m} / \mathrm{s})$ at $10 \mathrm{~km}$ Range, $\max =47.03$

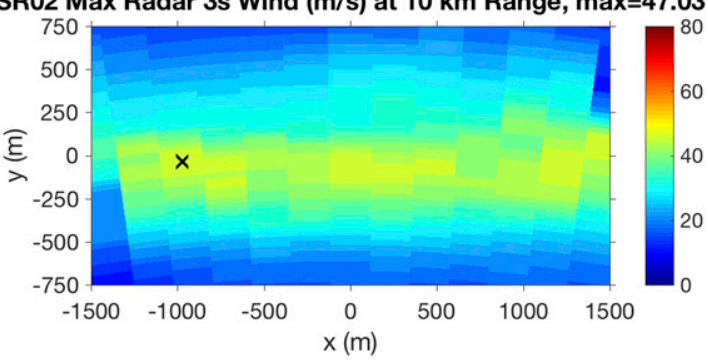

FIG. 6. As in Fig. 4, but for simulation SR02.

is a few meters at most and is negligible relative to the size of the radar beam. Therefore, for simplicity, we estimate the beam center height as

$$
h(r)=h_{0}+r \tan \phi .
$$

Since we are only concerned with the surface winds, $\phi=0.5^{\circ}$ is used here. It should be remembered that at this low angle, actual radar observations may be heavily contaminated by clutter as well as scattering/reflection of the lower part of the beam [i.e. where $\theta_{v i}$ in (5) is less than $-0.5^{\circ}$ ] in by the ground (Snyder et al. 2015). Even though there is no such direct interaction between the beam and the model surface in these experiments, the lack of observations below the surface shifts the centroid 
(a)

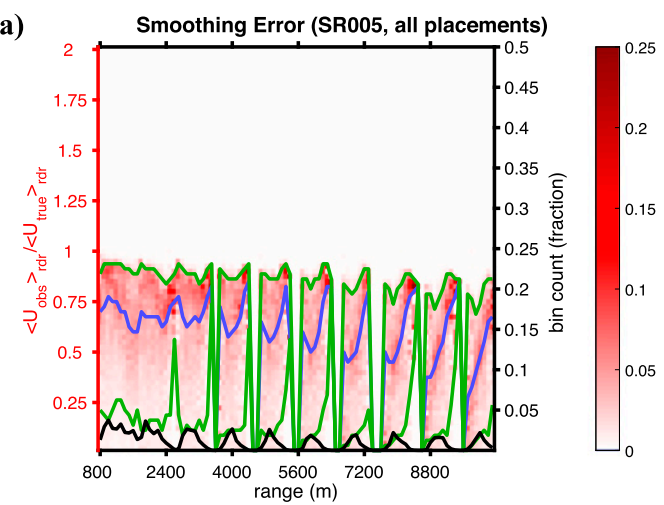

(c)

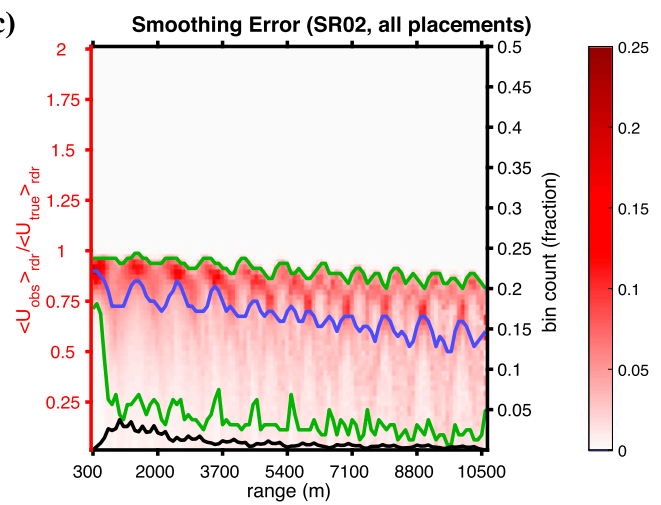

(b)

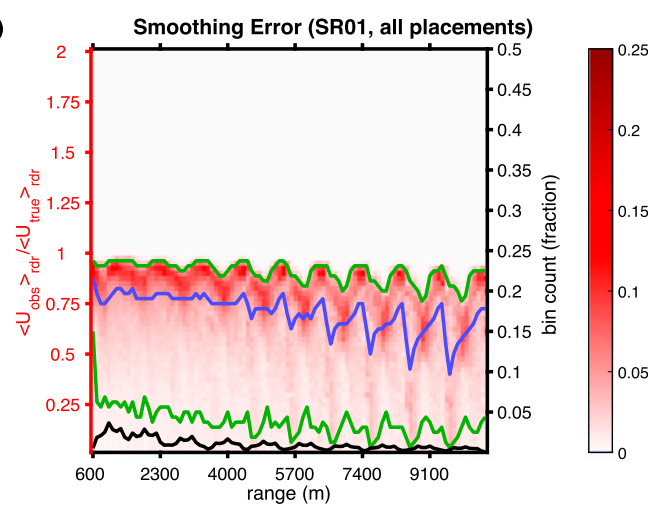

(d)

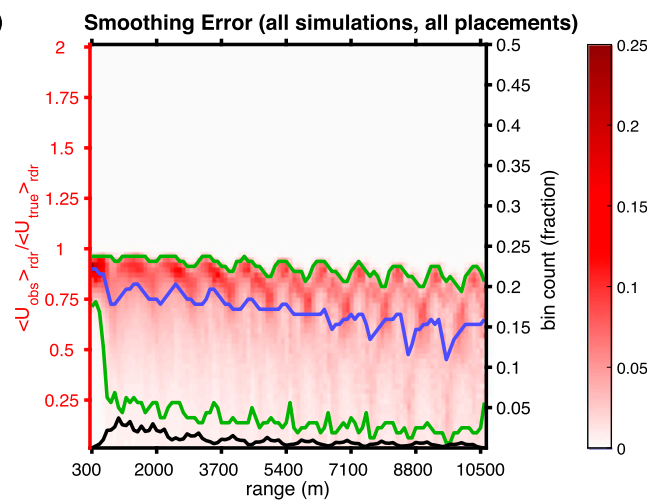

FIG. 7. Bivariate frequency diagrams of smoothing error as a function of range, aggregated over all radar placements, for simulation (a) SR005, (b) SR01, (c) SR02, and (d) all simulations. Median values for each wind speed bin are plotted in blue, 5th and 95th percentiles are plotted in green, and the fraction of the total count contained in each bin is plotted in black.

of the beam power distribution given in (5) upward, thereby increasing the effective beam center height. However, under these idealized conditions, this upward shift is on the order of $0.01^{\circ}$, which is also negligible relative to the size of the radar beam.

\section{c. Evaluating observation errors}

Evaluating sources of error requires clear, appropriate definitions of "truth." In the context of using radar observations to estimate the damage potential of tornadic winds, we deem that the goal is to use the radar wind observation at a given location to estimate the EF scale winds occurring there. In other words, we seek to use radial wind measurements for a resolution volume $V$ centered at $(x, y, z)$ with spherical dimensions $(\delta r, r \delta \theta, r \delta \phi)$ to estimate the maximum wind speed occurring within a horizontal area $A$ centered at $(x, y, 10 \mathrm{~m})$ with cylindrical dimensions $(\delta r, r \delta \theta)$, as illustrated in Fig. 1. We therefore obtain the observed instantaneous wind speed within $V\left(U_{\text {obs }}\right)$, the true maximum instantaneous radial wind speed within $V\left(U_{\text {true }}\right)$, the true maximum instantaneous horizontal wind speed within $V\left(S_{\text {true }}\right)$, and the true maximum instantaneous horizontal wind speed within $A\left(\mathrm{~S} 10_{\text {true }}\right)$.
After making the assumptions described in the previous section, we expect at the outset that the most significant remaining sources of error in the instantaneous radar observations will be smoothing due to averaging within the resolution volume, differences in direction between the true winds and the radar beam (since any cross-beam component of the wind is not observable), and the resolution volume elevation above or below the target level of $10 \mathrm{~m}$ AGL. Furthermore, the EF scale does not relate the damage severity to the maximum instantaneous $10-\mathrm{m}$ wind speed occurring at a given location, but rather to the maximum $10-\mathrm{m}, 3$-s gust ( $\mathrm{S} 10-3 \mathrm{~s})$ as noted, for example, in Marshall et al. (2014) and Snyder and Bluestein (2014). Snyder and Bluestein seek to account for this by averaging successive RaXPol measurements to estimate 2-s gusts within the $2013 \mathrm{El}$ Reno tornado. However, as they acknowledge, such averaging assumes that the wind speed varies linearly with time over the interval between successive scans; the validity of this assumption is unclear. Errors arising from this assumption and differences between the averaging period determined by the radar scans (e.g., $2 \mathrm{~s}$ for Snyder and Bluestein 2014) and the desired $3 \mathrm{~s}$ averaging period 
are combined here as a measure of error due to the temporal sampling interval.

Estimating the maximum $\mathrm{S} 10-3 \mathrm{~s}$ within $A$ by averaging the estimates of $\mathrm{S} 10$ obtained from $U_{\text {obs }}$ may also be erroneous because the location of the maximum instantaneous wind speed within $V$ may change substantially over the averaging time interval, particularly if $V$ is large. As a simple example, consider a $100 \mathrm{~m} \mathrm{~s}^{-1}$ gust moving through a resolution volume containing locations $\mathrm{A}$ and $\mathrm{B}$ and a background wind speed of $50 \mathrm{~m} \mathrm{~s}^{-1}$, such that the local wind speed at location A decreases linearly from $100 \mathrm{~m} \mathrm{~s}^{-1}$ at $t=0$ to $50 \mathrm{~m} \mathrm{~s}^{-1}$ at $t=3 \mathrm{~s}$ while the wind speed at location B increases linearly from $50 \mathrm{~m} \mathrm{~s}^{-1}$ at $t=0$ to $100 \mathrm{~m} \mathrm{~s}^{-1}$ at $t=3 \mathrm{~s}$. S10 (the maximum wind speed anywhere in the volume) is $100 \mathrm{~m} \mathrm{~s}^{-1}$ throughout the averaging period, so the estimate of S10-3s obtained by averaging $\mathrm{S} 10$ is $100 \mathrm{~m} \mathrm{~s}^{-1}$; however, the true value of S10-3s (i.e., the maximum local 3-s gust at any fixed point in the volume, either location $\mathrm{A}$ or location $\mathrm{B}$ ), is $75 \mathrm{~m} \mathrm{~s}^{-1}$. This disparity is hereafter referred to as representativeness error.

In light of these considerations, we evaluate the individual error sources and the total error in estimating the maximum S10-3s within each resolution volume as ratios:

$$
\begin{aligned}
& E_{\mathrm{smth}}=\left\langle U_{\mathrm{obs}}\right\rangle_{\mathrm{rdr}} /\left\langle U_{\text {true }}\right\rangle_{\mathrm{rdr}} \quad \text { (smoothing error), } \\
& E_{\text {dir }}=\left\langle U_{\text {true }}\right\rangle_{\text {rdr }} /\left\langle S_{\text {true }}\right\rangle_{\text {rdr }} \quad \text { (direction error), } \\
& E_{\text {elev }}=\left\langle S_{\text {true }}\right\rangle_{\text {rdr }} /\left\langle\mathrm{S} 10_{\text {true }}\right\rangle_{\text {rdr }} \quad \text { (elevation error), } \\
& E_{\text {tem }}=\left\langle\mathrm{S} 10_{\text {true }}\right\rangle_{\text {rdr }} /\left\langle\mathrm{S} 10_{\text {true }}\right\rangle_{3 \mathrm{~s}} \quad \text { (temporal sampling } \\
& \text { error), } \\
& E_{\text {rep }}=\left\langle\mathrm{S} 10_{\text {true }}\right\rangle_{3 \mathrm{~s}} / \mathrm{S} 10-3 \mathrm{~s} \text { (representativeness error), } \\
& E_{\mathrm{tot}}=\left\langle U_{\mathrm{obs}}\right\rangle_{\mathrm{rdr}} / \mathrm{S} 10-3 \mathrm{~s} \quad \text { (total error). }
\end{aligned}
$$

Angle brackets denote temporal averages, with \langle\rangle$_{\text {rdr }}$ indicating an average obtained from successive radar observation times over the maximum possible period $\leq 3 \mathrm{~s}$ [e.g., at times $\mathrm{t} 0$ and $\mathrm{t} 0+2 \mathrm{~s}$ for the method in Snyder et al. (2015)] and \langle\rangle$_{3 \mathrm{~s}}$ indicating a "true" 3-s average obtained using the $10-\mathrm{Hz}$ model output at $10 \mathrm{~m}$ AGL.

We examine the distribution of the errors detailed in (8a)-(8f) using bivariate frequency diagrams. The error is binned on the $y$ axis, while a variable that may be estimated a priori and may reasonably be expected to correlate with the error is binned on the $x$ axis. The range from the radar to the target seems to be a suitable $x$ variable; for example, greater range means a larger resolution volume (and hence more potential smoothing of the wind field) with a greater extent in altitude (and hence more potential error due to elevation). The observed wind speed $\left(U_{\mathrm{obs}}\right)$ is another possibility, since one might speculate that a location with strong observed winds may be more likely to have
TABLE 1. Radar-estimated and true global maximum 10-m, 3-s gusts. Global maximum $10-\mathrm{m}, 3-\mathrm{s}$ gusts estimated from $0.5-\mathrm{Hz}$ radar scans at various placements ("observed max") compared with true global maxima obtained from $10-\mathrm{Hz}$ model output (with "true max S10-3s" shown in italics). All values are in $\mathrm{ms}^{-1}$. Corresponding EF scale ratings are given in parentheses.

\begin{tabular}{lccc}
\hline \hline & SR005 & SR01 & SR02 \\
\hline 1 km south observed max & $59.2(\mathrm{EF} 2)$ & $78.7(\mathrm{EF} 4)$ & $63.3(\mathrm{EF} 3)$ \\
$2 \mathrm{~km}$ south observed max & $55.4(\mathrm{EF} 2)$ & $68.0(\mathrm{EF} 3)$ & $59.6(\mathrm{EF} 2)$ \\
$3 \mathrm{~km}$ south observed max & $51.8(\mathrm{EF} 2)$ & $61.5(\mathrm{EF} 3)$ & $58.2(\mathrm{EF} 2)$ \\
$4 \mathrm{~km}$ south observed max & $51.5(\mathrm{EF} 2)$ & $57.7(\mathrm{EF} 2)$ & $56.8(\mathrm{EF} 2)$ \\
$5 \mathrm{~km}$ south observed max & $52.2(\mathrm{EF} 2)$ & $53.8(\mathrm{EF} 2)$ & $55.1(\mathrm{EF} 2)$ \\
$6 \mathrm{~km}$ south observed max & $47.5(\mathrm{EF} 1)$ & $54.1(\mathrm{EF} 2)$ & $49.9(\mathrm{EF} 2)$ \\
$7 \mathrm{~km}$ south observed max & $41.6(\mathrm{EF} 1)$ & $51.5(\mathrm{EF} 2)$ & $48.9(\mathrm{EF} 1)$ \\
$8 \mathrm{~km}$ south observed max & $40.9(\mathrm{EF} 1)$ & $48.2(\mathrm{EF} 1)$ & $47.9(\mathrm{EF} 1)$ \\
$9 \mathrm{~km}$ south observed max & $38.4(\mathrm{EF} 1)$ & $45.9(\mathrm{EF} 1)$ & $50.0(\mathrm{EF} 2)$ \\
$10 \mathrm{~km}$ south observed max & $36.6(\mathrm{EF} 0)$ & $45.6(\mathrm{EF} 1)$ & $47.0(\mathrm{EF} 2)$ \\
True max S10-3s & $75.0(\mathrm{EF} 4)$ & $85.3(\mathrm{EF} 4)$ & $78.0(\mathrm{EF} 4)$ \\
\hline
\end{tabular}

large errors [particularly with respect to (8c)-(8f), because of increased near-surface shear and the possibility of unresolved subvortices]. However, such a correlation would obviously be affected by the direct impact of the radar beam direction on $U_{\text {obs }}$. Furthermore, for an intense vortex, the deviation between the radar beam direction and the true wind direction may have secondary impacts that further complicate matters; for example, with an intense vortex, there is a strong gradient in $U_{\text {obs }}$ where the beam direction lies at a large angle to the true wind direction, which would be expected to increase $E_{\text {smth }}$ in (8a). Therefore, we also examine frequency diagrams of the errors as functions of a simple prediction of the deviation between the radar beam direction and the true wind direction (hereafter "deviation from beam," with "dev pred" denoting the value predicted from observations); the derivation of this prediction is described in the appendix.

To focus on conditions within the vortex, the plotted results are limited to the continuous region where the maximum observed wind speed at a given radius from the estimated vortex center is $\geq 20 \mathrm{~m} \mathrm{~s}^{-1}$ after a $200 \mathrm{~m} \times$ $200 \mathrm{~m}$ top-hat filter is applied. To construct the bivariate histograms, the error results (again, expressed as ratios) are binned on the $y$ axis in increments of 0.025 . On the $x$ axis, distances from the radar are binned in $100-\mathrm{m}$ increments, $U_{\text {obs }}$ values are binned in $5 \mathrm{~m} \mathrm{~s}^{-1}$ increments, and $\operatorname{dev}_{\text {pred }}$ values are binned in $5^{\circ}$ increments. To keep the figures compact and focused on sufficiently large sample sizes, the diagram domains extend only between the minimum and maximum $x$ bins with counts $n \geq 100$. To further elucidate the distribution of data points, the diagrams include line plots showing the median (blue), 5th percentile (green, lower), 95th percentile (green, upper), and fraction of the total number of samples contained in each $x$ bin (black). 
(a)

(a)

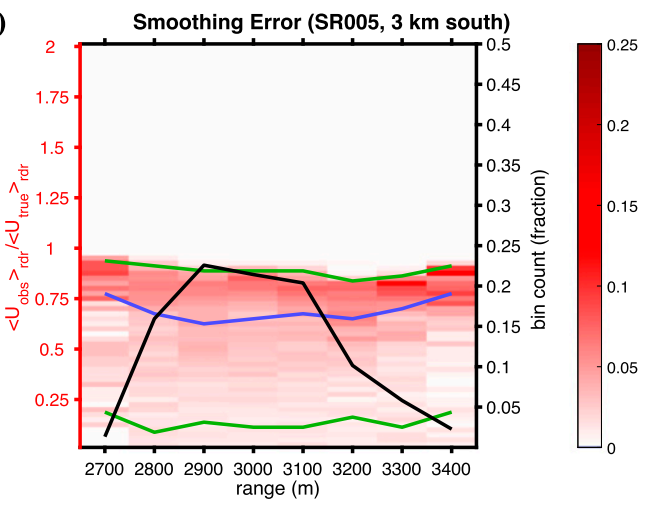

(c)

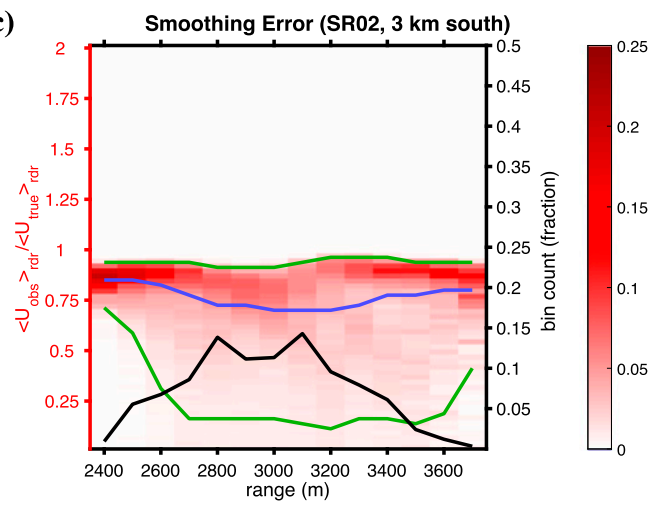

(b)

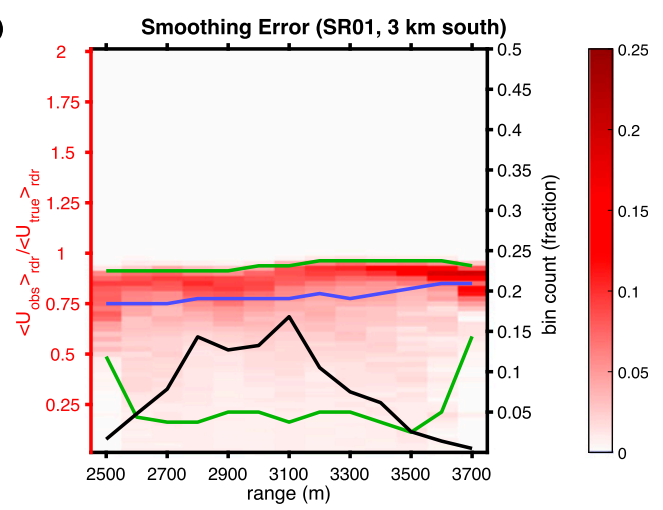

(d)

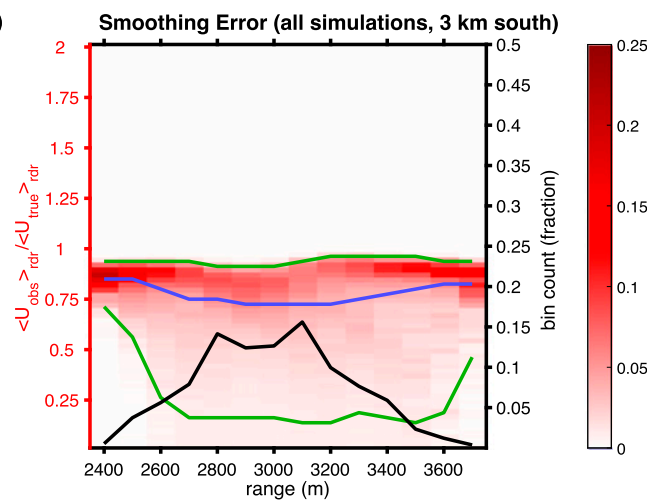

FIG. 8. As in Fig. 7, but only for radar placement $3 \mathrm{~km}$ south of the vortex track.

\section{Results}

\section{a. Temporal sampling and representativeness error}

Figure 2 shows frequency diagrams of $E_{\text {tem }}$ aggregated over all radar placements for each of the simulations, as well as the combined result over all simulations, as functions of $U_{\text {obs. }}$. The diagrams indicate that this error source is negligible for the cases studied here, accounting for an adjustment of $<5 \%$ in the vast majority of instances; the distribution is so compact that the lines indicating the median and 5th and 95th percentiles are often difficult to decipher. This holds true even for higher values of $U_{\mathrm{obs}}$, which constitute a small fraction of the measurements. It also holds true if $E_{\mathrm{tem}}$ is instead plotted as a function of radar range or $\operatorname{dev}_{\text {pred }}$ (not shown).

Likewise, the frequency diagrams of $E_{\text {rep }}$ as a function of $U_{\text {obs }}$ (Fig. 3) indicate a generally negligible source of error. The median value of $E_{\text {rep }}$ increases slightly at higher values of $U_{\text {obs }}$ for the low-swirl vortex (SR005), perhaps because the simulated low-swirl vortex center is more prone to deviant motion [suggested by the momentum budget results in Nolan et al. (2017)]. However, even here, the combined effect of $E_{\text {tem }}$ and $E_{\text {rep }}$ is in the range of $5-10 \mathrm{~m} \mathrm{~s}^{-1}$ at most, which is comparable to the uncertainty commonly assigned to actual mobile Doppler measurements of strong tornadoes due to increased spectrum width (e.g., see Bluestein et al. 1993; Wurman et al. 2007). While these results do not extend to the most extreme cases [e.g., small subvortices with wind speeds exceeding $100 \mathrm{~m} \mathrm{~s}^{-1}$ translating rapidly around the main vortex as described by Wurman et al. (2014), Snyder and Bluestein (2014), and Wakimoto et al. (2015)], it appears that Snyder and Bluestein's (2014) method of approximating the 3-s gust by averaging successive radar observations $2 \mathrm{~s}$ apart would work very well here if other error sources were removed. Therefore, for the remaining analysis, $E_{\mathrm{tem}}$ and $E_{\mathrm{rep}}$ are ignored.

\section{b. Smoothing and direction error}

The effect of beam broadening on the wind observations is partially illustrated by the wind swaths in Figs. 4-6. In accordance with general understanding and prior studies [e.g., see Figs. 1 and 4 of Wood and Brown (1997)], larger-resolution volumes at longer ranges result in increased smoothing and deterioration of both structural detail and estimates of local maxima. Furthermore, the location of the observed global maximum can shift dramatically depending on radar position. 
(a)

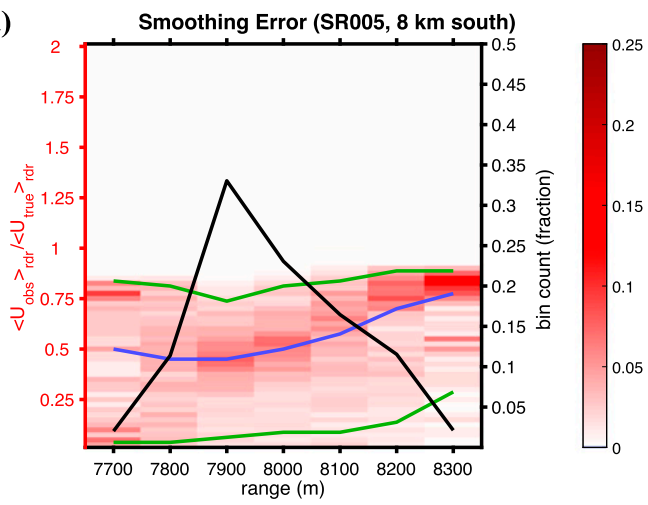

(c)

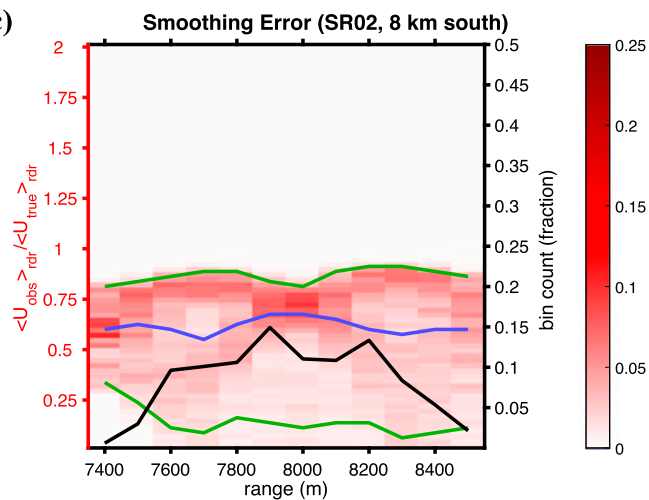

(b)

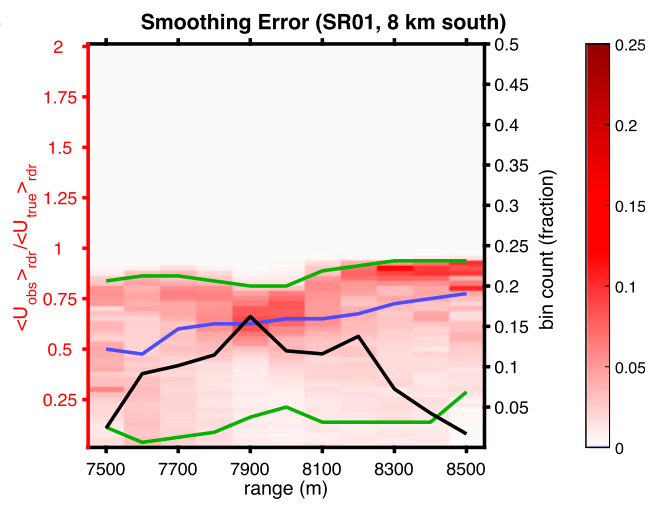

(d)

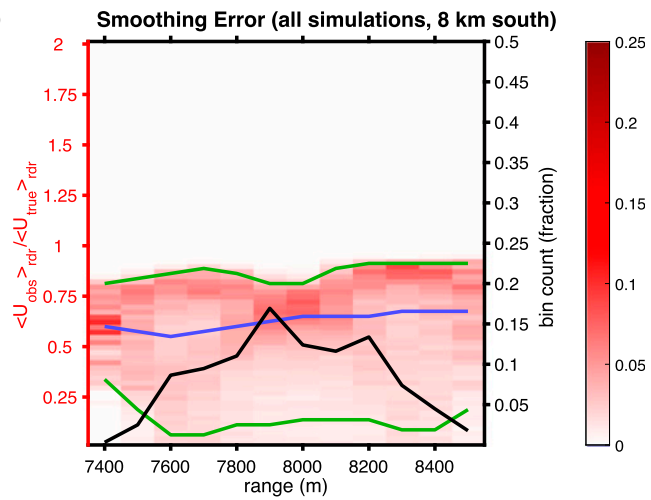

FIG. 9. As in Fig. 7, but only for radar placement $8 \mathrm{~km}$ south of the vortex track.

However, the relationship between radar range and $E_{\text {smth }}$ is more complex than one might expect. The data in Fig. 7 are aggregated over all radar placements from $1 \mathrm{~km}$ south to $10 \mathrm{~km}$ south in an effort to obtain a large sample set in which the ranges from successive radar placements overlap to provide a continuous diagram of $E_{\text {smth }}$ as a function of radar range, although the narrowness of the SR005 vortex (see Fig. 4) does not allow for such an overlap at longer ranges and produces the gaps shown in Fig. 7a. All of the plots show a general increase in smoothing with range as a downward trend in median $E_{\text {smth }}$ (decreasing on average from approximately $0.75-0.85$ for the $1 \mathrm{~km}$ south radar placement to approximately $0.4-0.6$ for the $10 \mathrm{~km}$ south radar placement), which is more pronounced in the low-swirl case. However, there is a great deal of variability, with the 5th and 95th percentiles spanning much of the interval from 0 to 1 throughout the experiments. Also, the minimum median $E_{\text {smth }}$ (i.e., the greatest amount of smoothing) for a given radar placement is not generally found for the observations that are farthest away; as highlighted in Figs. 8 and 9 , the median values of $E_{\text {smth }}$ for the shortest and longest observed ranges are similar to each other when the radar is placed near the vortex track (e.g., $3 \mathrm{~km}$ south in Fig. 8), and the minimum median $E_{\text {smth }}$ is actually found near the point of closest approach when the radar is placed farther from the vortex track (e.g., $8 \mathrm{~km}$ south in Fig. 9).

This result seems counterintuitive until one considers the effect of wind direction relative to the radar beam. Since the strongest winds are expected to be on the right side of the vortex and directed parallel to the vortex translation, it is also expected that those winds will be directed largely perpendicular to the radar beam at the time of closest approach in the scenarios tested here. Because of the curved flow around the vortex, an enhanced gradient in $U_{\text {obs }}$ exists in the same area, and this increased gradient can in turn increase the spatial smoothing that occurs within a given resolution volume.

The role played by the deviation from beam is confirmed by the diagrams in Fig. 10. The distribution broadens as the deviation from beam increases, due to increased errors in $\operatorname{dev}_{\text {pred }}$ (see the appendix) as well as a greater sensitivity of $\left\langle U_{\text {true }}\right\rangle_{\text {rad }}$ (i.e., the time-averaged maximum true Doppler wind speed in a given resolution volume) to small fluctuations when $U_{\text {obs }}$ is small. Even so, $E_{\text {smth }}$ shows a much more well-defined relationship with $\operatorname{dev}_{\text {pred }}$ (with the median of $E_{\text {smth }}$ varying smoothly from approximately 0.85 for small $\mathrm{dev}_{\text {pred }}$ to 0.5 or less for large $\left.\operatorname{dev}_{\text {pred }}\right)$. Also, a comparison of Figs. 8 and 9 
(a)

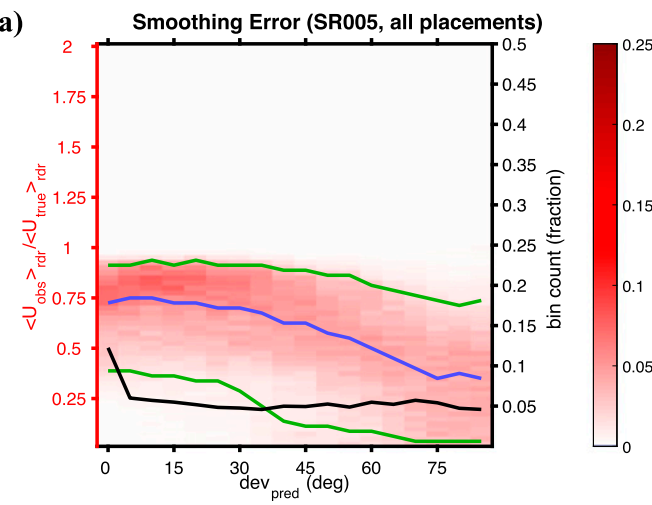

(c)

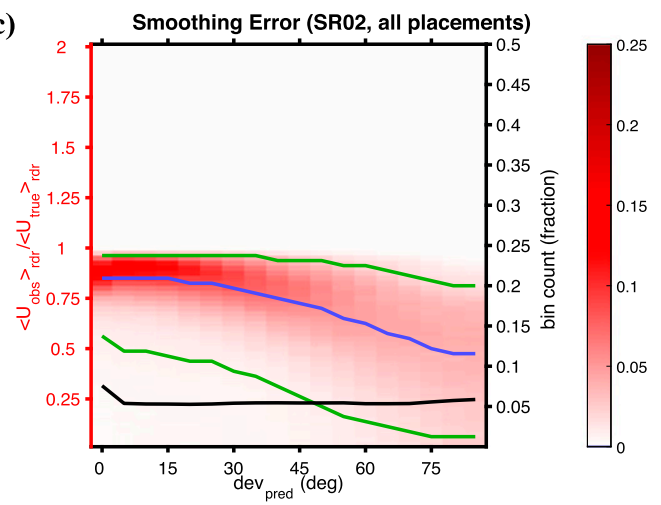

(b)

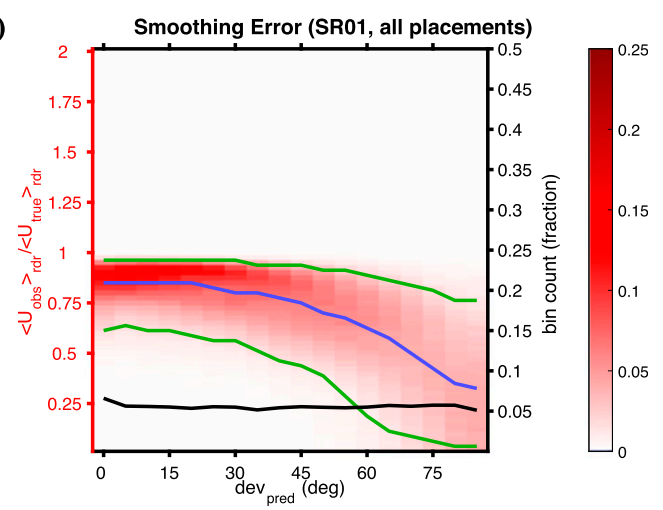

(d)

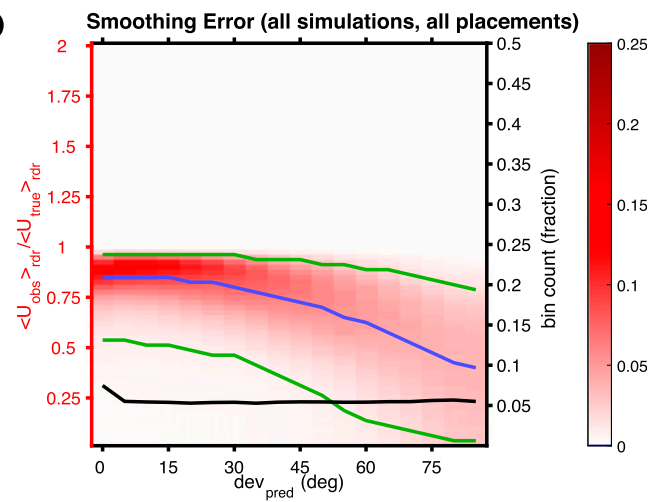

FIG. 10. As in Fig. 7, but plotted as a function of predicted deviation from beam.

suggests that this effect becomes more prominent when the radar is placed farther from the track; Fig. 8 shows a reduction in smoothing (i.e., an increase in $E_{\text {smth }}$ ) near the point of closest approach, hinting that the impact of reduced-resolution volume size at smaller range remains discernible relative to the impact of the deviation from beam for the $3-\mathrm{km}$ radar placement, but no such reduction appears in Fig. 9 for the 8-km radar placement. (On the other hand, it should be noted that the evaluation of $d v_{\text {pred }}$ becomes less reliable, and thus the effect of the deviation from beam becomes more difficult to evaluate accurately, if the range is large enough that the vortex itself is poorly resolved by the radar. For example, compare the distributions from the $1 \mathrm{~km}$ south and $10 \mathrm{~km}$ south radar placements for the medium-swirl vortex shown in Fig. 11.)

The smoothing error $E_{\text {smth }}$ is diagrammed as a function of $U_{\text {obs }}$ in Fig. 12. While this relationship is also more consistent than the one for radar range, there is a good deal of variation from simulation to simulation, particularly when comparing the low-swirl vortex to the medium-swirl and high-swirl vortices. Moreover, this result is somewhat redundant; the best correlation appears where $U_{\text {obs }}$ is large, which will generally correspond to a small $\operatorname{dev}_{\text {pred }}$. Likewise, the greatest spread occurs where $U_{\text {obs }}$ is small, which (in a vortex) will generally correspond to where $\operatorname{dev}_{\text {pred }}$ is large. This is confirmed in Fig. 13, which shows a similar relationship between $E_{\text {dir }}$ and $U_{\text {obs }}$ even though ( $\left.8 \mathrm{~b}\right)$ provides no basis for such a relationship.

These results suggest that $E_{\text {smth }}$ is substantially influenced in these cases by both the range and the deviation from beam, with the influence of the deviation from beam increasingly predominating at longer ranges. Since $E_{\text {dir }}$ is basically a function of deviation from beam alone, one would expect the $E_{\text {dir }}$ plots to also show a strong dependence on $\operatorname{dev}_{\text {pred }}$, assuming that $\operatorname{dev}_{\text {pred }}$ reasonably approximates the true deviation from beam. Figure 14 shows that the distributions for $E_{\text {dir }}$ as a function of $d^{2} v_{\text {pred }}$ are indeed quite similar in shape to the distributions for $E_{\mathrm{sm} t h}$, although $E_{\mathrm{dir}}$ is typically very close to unity (indicating very small errors) for $\operatorname{dev}_{\text {pred }}<$ $30^{\circ}$. Also, Fig. 15 shows that the $E_{\text {dir }}$ distribution at longer range is surprisingly compact, particularly for large $\operatorname{dev}_{\text {pred. }}$. While much of this is due to the deviation from beam estimate becoming less accurate at long ranges for the smaller vortices, a reexamination of Figs. 2-4 suggests that it also partially stems from the fact that placing the radar closer to the track aligns the beam more with the vortex motion early and late in 
(a)

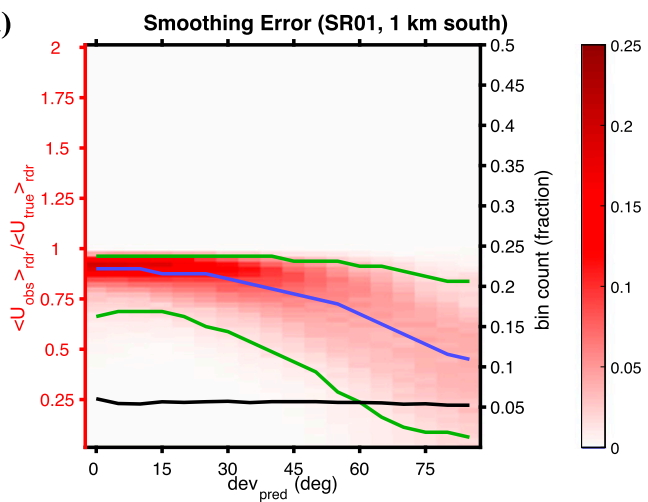

(b)

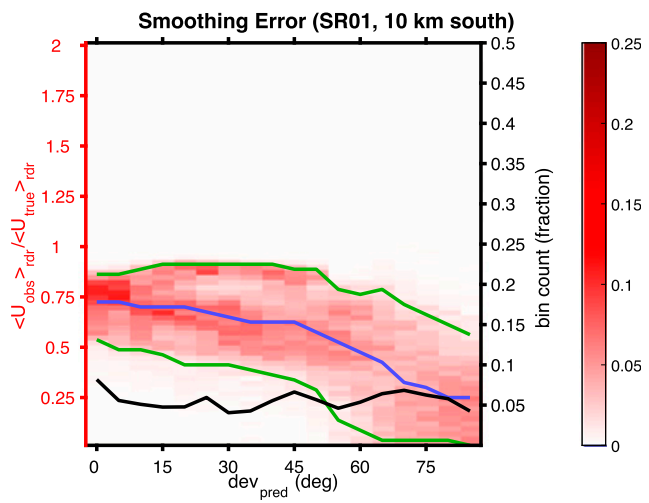

FIG. 11. As in Fig. 10, but only for simulation SR01 for radar placements (a) 1 and (b) $10 \mathrm{~km}$ south of the vortex track.

the observation period. While this is more likely to produce a good measurement of the maximum wind speed (i.e., typically located in the right-front quadrant) at those times, it simultaneously degrades the measurement of strong inflow bands (e.g., cf. the upper-left corners of Figs. $6 b$ and $6 c$ ) and leaves a more persistent "void" in the middle of the track since the winds ahead of and behind the vortex center are largely perpendicular to the beam. (Note the much weaker radial winds near the track centers on the far-right sides of
Figs. $4 b, 5 b$, and $6 b$.) Thus, the proportion of large$\operatorname{dev}_{\text {pred }}$ observations with large errors is increased when the radar is located closer to the vortex track.

\section{c. Elevation error}

Figure 16 diagrams the distribution of $E_{\text {elev }}$ as a function of radar range. It should be borne in mind that, with the elevation angle and beamwidth used here, the bottom of the resolution volume is essentially at ground level and the top of the resolution volume extends above (a)

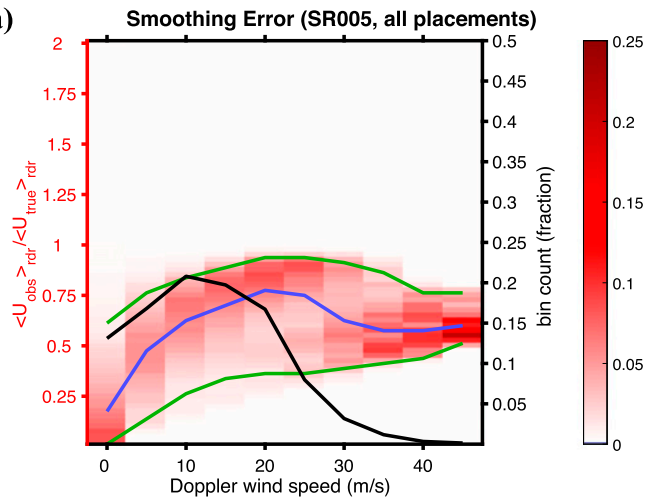

(c)

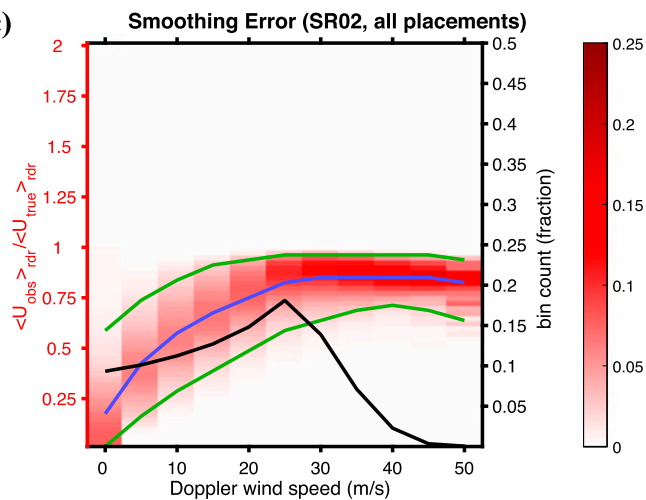

(b)

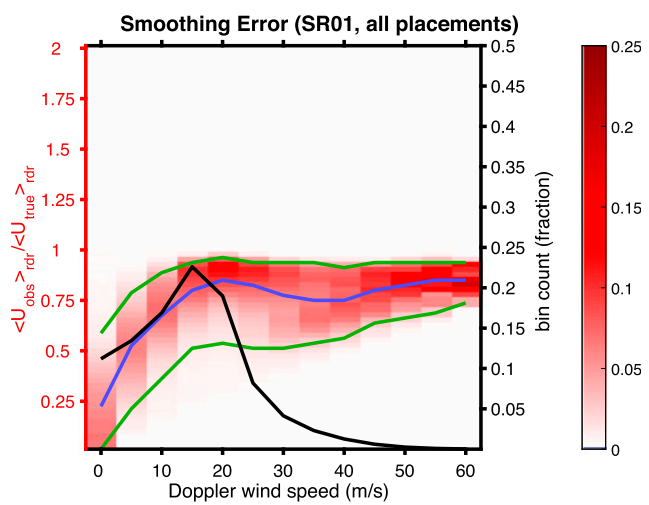

(d)

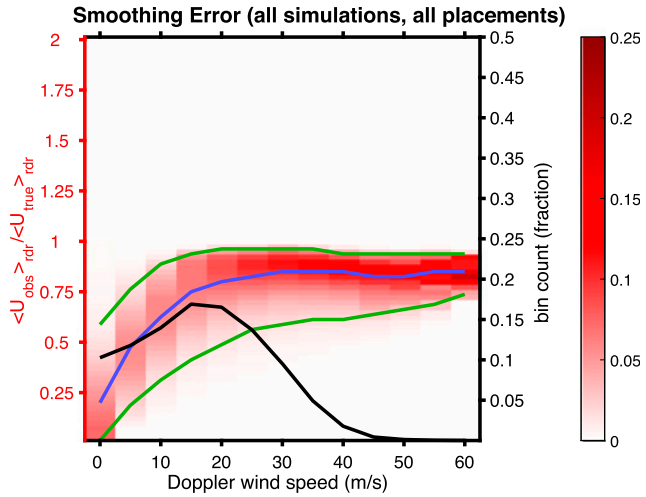

FIG. 12. As in Fig. 7, but as a function of observed Doppler wind speed. 
(a)

a)

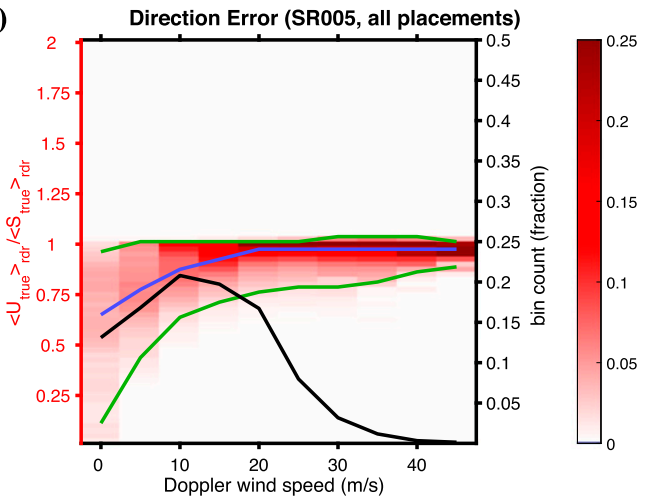

(c)

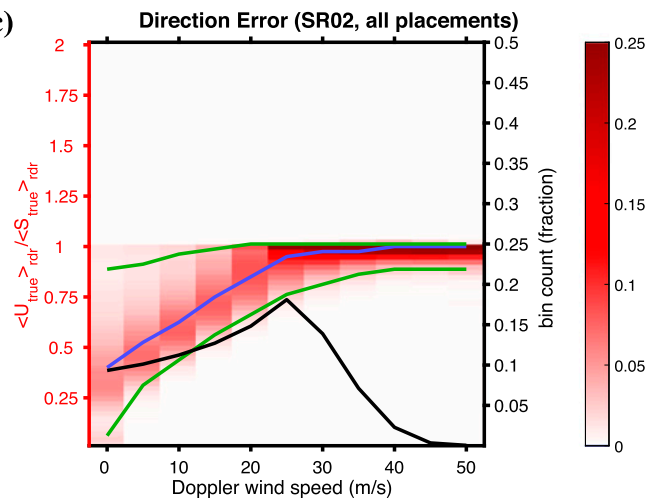

(b)

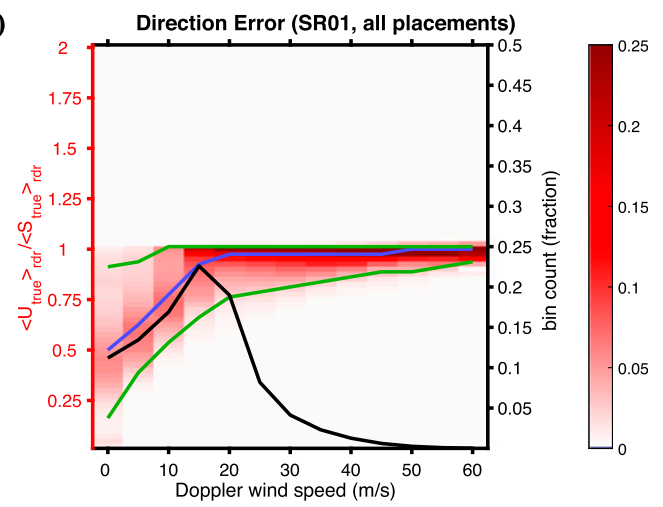

(d)

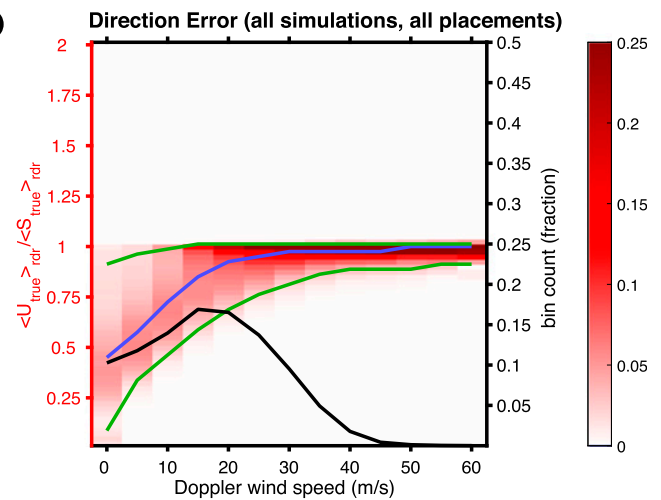

FIG. 13. As in Fig. 7, but for direction error as a function of observed Doppler wind speed.

$10 \mathrm{~m}$ AGL over nearly all of the ranges plotted. Therefore, the resolution volume generally encompasses the target $10-\mathrm{m}$ altitude; since the numerator in $(8 \mathrm{c})$ is the maximum horizontal wind speed occurring anywhere in the volume, $E_{\text {elev }}$ is equal to 1 if the maximum horizontal wind speed occurs at $10 \mathrm{~m}$ AGL and generally greater than 1 if it occurs at any other height (whether above or below $10 \mathrm{~m}$ ). The exception is seen in Fig. 16c, where a portion of the broader high-swirl vortex is sampled at a range of less than $\sim 500 \mathrm{~m}$ by the $1 \mathrm{~km}$ south radar placement; in that circumstance, the entire resolution volume is below $10 \mathrm{~m} \mathrm{AGL}$ and it is therefore possible (since the target $10 \mathrm{~m}$ altitude is not included in the samples) for $E_{\text {elev }}$ to be less than 1. Otherwise, it appears that $E_{\text {elev }}$ is an overestimate that serves to counteract the underestimates expressed by $E_{\mathrm{smth}}$ and $E_{\mathrm{dir}}$.

Figure 16 shows that the sensitivity of $E_{\text {elev }}$ to the range is not the same in all cases; the median value trends upward to approximately 1.2 at the $10-\mathrm{km}$ range for the low-swirl and high-swirl vortices but only up to approximately 1.1 for the medium-swirl vortex. This can largely be explained by examining the time-mean structure of the vortices. Figure 17 shows vertical cross sections of horizontal and vertical wind speed taken through the vortex centers in the positive $x$ direction (i.e., in the approximate direction of vortex motion over the period of observation). All three vortices have an elevated horizontal wind speed maximum on the leading edge extending $>100 \mathrm{~m}$ AGL, but the near-surface intensity varies considerably. There is no near-surface time-mean maximum in the low-swirl vortex; this is similar to previous low-swirl vortex simulations [e.g., see Fig. 13 in Rotunno (2013)], although, as noted in Dahl et al. (2017), it contrasts with the strong near-surface maximum in the stationary SR005 vortex shown in Nolan et al. (2017) because of the reduction in effective swirl due to translation (Lewellen et al. 2000). Because the time-mean maximum wind speed is located well above the surface, the median $E_{\text {elev }}$ increases with range (as the resolution volume spreads vertically) before leveling off for ranges $>6 \mathrm{~km}$ (where the resolution volume extends $>100 \mathrm{~m}$ AGL and contains the elevated maximum).

Similarly, while the high-swirl vortex has a local timemean horizontal wind speed maximum nearer to the ground, centered at roughly $30 \mathrm{~m}$ AGL, it is not as intense as the elevated maximum (not shown). Thus, while the median $E_{\text {elev }}$ at the medium range is not quite as large as in the case of the low-swirl vortex, there is a steady upward trend that persists to a longer range than 
(a)

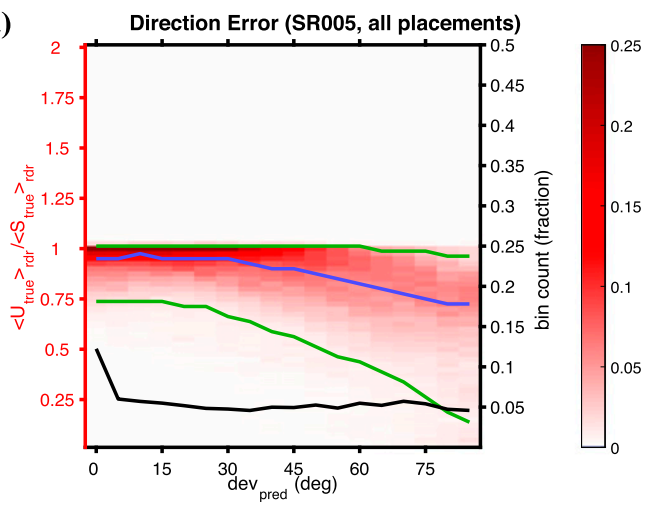

(c)

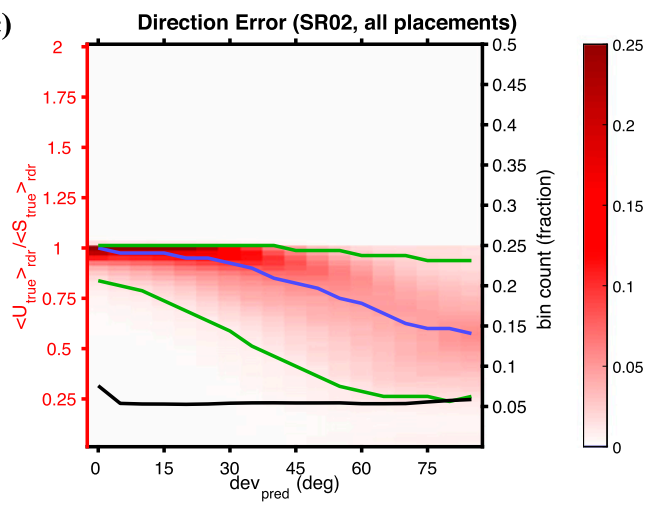

(b)

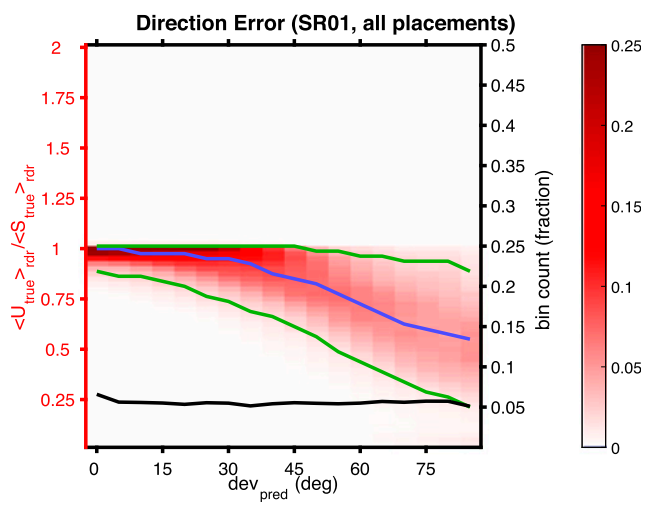

(d)

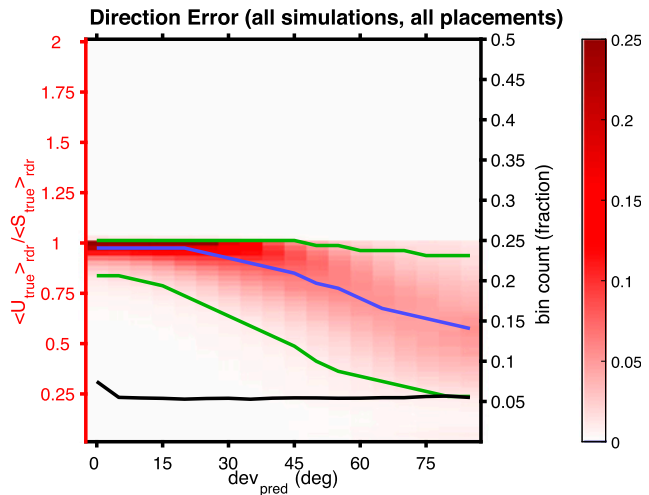

FIG. 14. As in Fig. 13, but plotted as a function of predicted deviation from beam.

the trend for the low-swirl vortex does (since the elevated maximum for the high-swirl vortex is also broader), yielding a similar value at the $10-\mathrm{km}$ range. In contrast, the near-surface time-mean maximum for the medium-swirl vortex, which is centered $\sim 15-20 \mathrm{~m}$ AGL, is more intense than the elevated maximum; since this level is contained in the resolution volume at all ranges $>1 \mathrm{~km}$, the median $E_{\text {elev }}$ is noticeably less sensitive to increased range in this case, although a small increase is still seen as a result of the increased resolution volume size providing a greater chance of sampling local wind speed fluctuations at other elevations that exceed the near-surface maximum at the time of observation.

There are a couple of side points to note regarding the vertical structure of the vortices. First, since the radar beam is not strictly horizontal, the possibility of the vertical velocity "contaminating" radar observations that are intended to represent the horizontal winds should be considered. Figure 18 shows time-mean maximum (a)

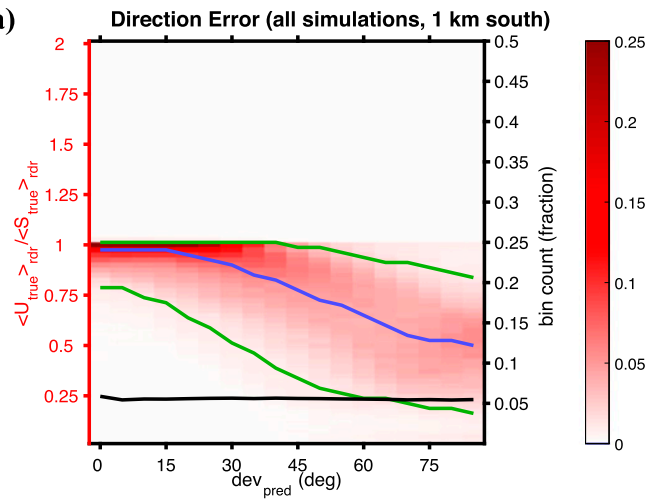

(b)

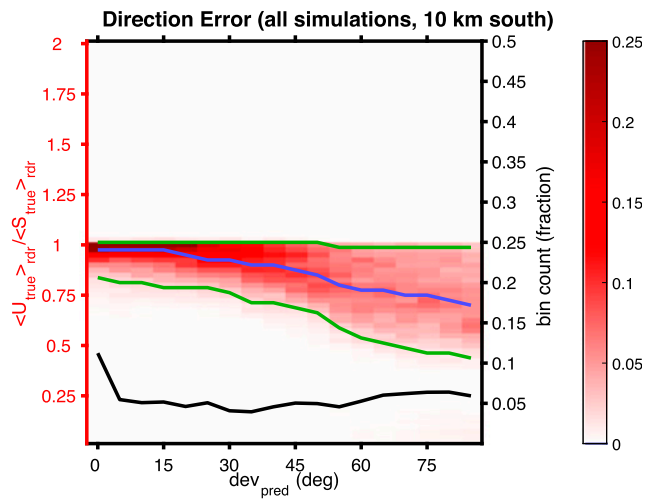

FIG. 15. As in Fig. 14, but for all simulations and only for radar placements (a) 1 and (b) $10 \mathrm{~km}$ south of the vortex track. 
(a)

(a)

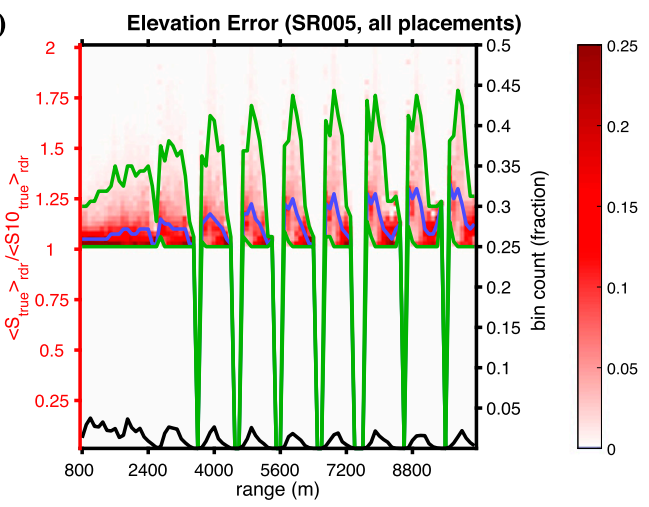

(c)

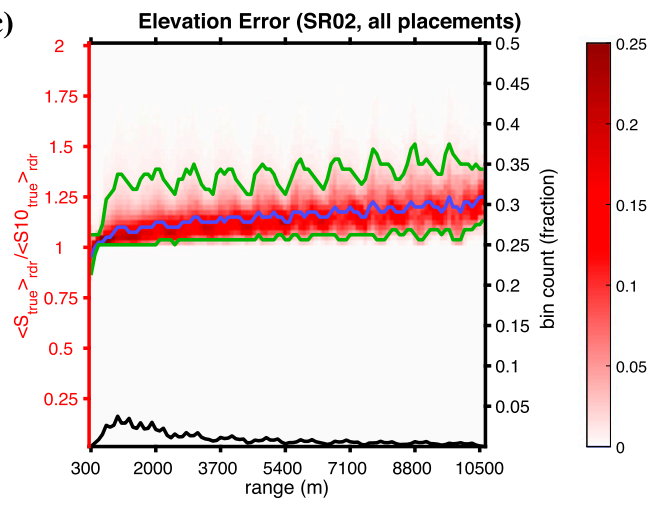

(b)

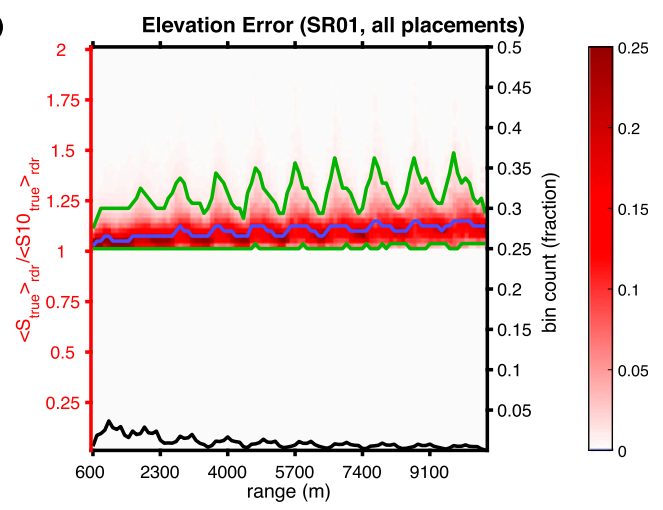

(d)

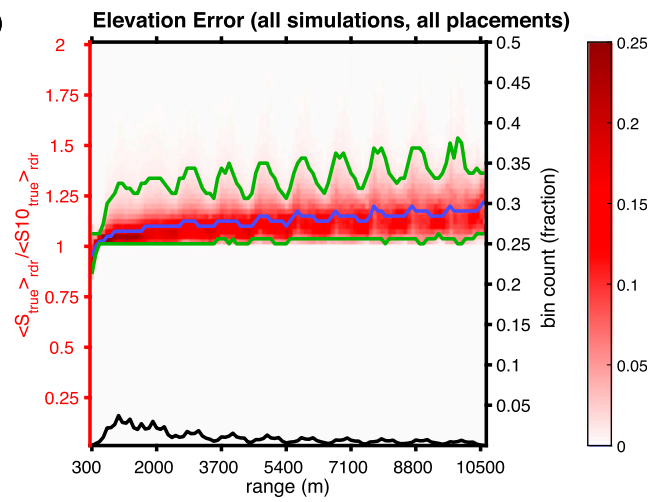

Fig. 16. As in Fig. 7, but for elevation error as a function of radar range.

vertical velocities peaking near $30 \mathrm{~m} \mathrm{~s}^{-1}$ for the low-swirl vortex and $20 \mathrm{~m} \mathrm{~s}^{-1}$ for the medium-swirl and high-swirl vortices, which amount to an adjustment of roughly $0.3-0.5 \mathrm{~m} \mathrm{~s}^{-1}$ when projected onto the $1^{\circ}$ elevation angle marking the approximate upper limit of the resolution volume. As was the case for the stationary vortices described in Nolan et al. (2017), intense local fluctuations (in the main vortex for the low-swirl case as well as in subvortices for the medium-swirl and high-swirl cases) occasionally produce updrafts briefly exceeding $100 \mathrm{~ms}^{-1}$; however, this still only amounts to an adjustment of around $2 \mathrm{~m} \mathrm{~s}^{-1}$ at a $1^{\circ}$ elevation angle, which is also likely to be substantially diminished by smoothing because of the small size of the features in question.

Second, as described previously, the altitude of the time-mean, near-surface horizontal wind speed maximum in these cross sections (when present) is roughly between 15 and $30 \mathrm{~m}$ AGL. This contrasts with recent radar observations of a tornado at extremely close range reported by Kosiba and Wurman (2013), which show the maximum wind speeds consistently occurring near $5 \mathrm{~m}$ AGL over the tornado's lifespan (exceeding the corresponding $10-\mathrm{m}$ wind speeds by a median value of $10 \%-$ $15 \%)$, as well as other reports of the strongest observed tornadic winds occurring at the lowest radar level (e.g.,
Wurman et al. 2007; Snyder et al. 2015). As discussed in section 1 , the variability of wind profiles in tornadic surface layers is not well understood, and the mixed nature of the observational record reflects that; for example, Fig. 4 of Wurman et al. (2007) illustrates a case in which the observed wind speed maxima typically occurred at $\sim 50 \mathrm{~m}$ AGL (the second-to-lowest level observed by the radar) over a 1-min observation period, as well as a case in which the maximum observed wind speed within the lowest $\sim 200 \mathrm{~m}$ AGL did not appear to vary much with height. Thus, it is difficult at present to know the significance of the apparent discrepancy between these simulations and observations like those reported in Kosiba and Wurman (2013). However, the possibility that the near-surface wind speeds in these simulations are artificially reduced to some degree by the nontrivial influence of the subgrid turbulence parameterization below $10 \mathrm{~m}$ AGL (described in section 2a) must be borne in mind.

\section{d. Total error}

Figures 18, 19, and 20 diagram the distribution of $E_{\text {tot }}$ as a function of radar range, $U_{\mathrm{obs}}$, and $\mathrm{dev}_{\text {pred }}$, respectively. They are quite similar to the corresponding $E_{\text {smth }}$ plots in Figs. 7, 10, and 9, respectively, suggesting that smoothing 
(a)

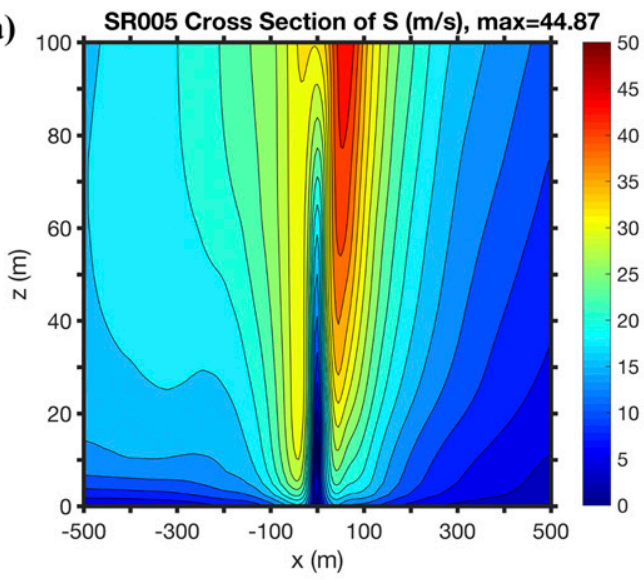

(c)

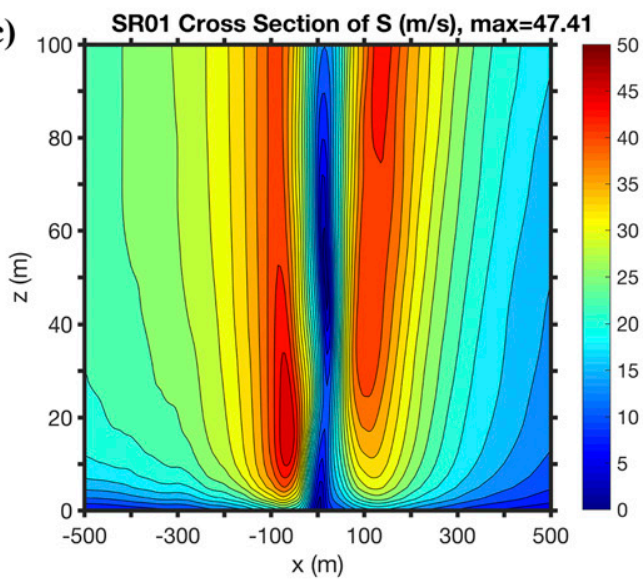

(e)

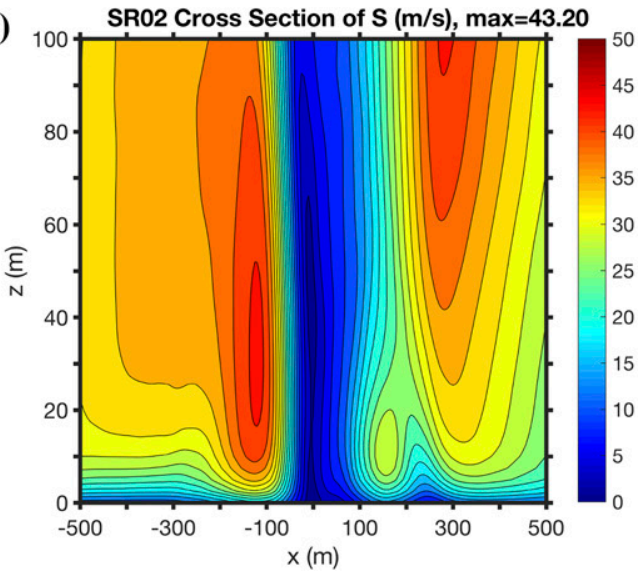

(b)

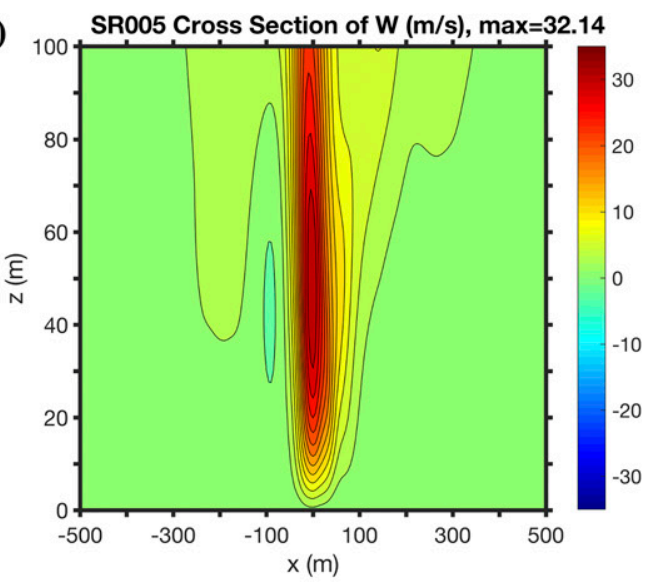

(d)

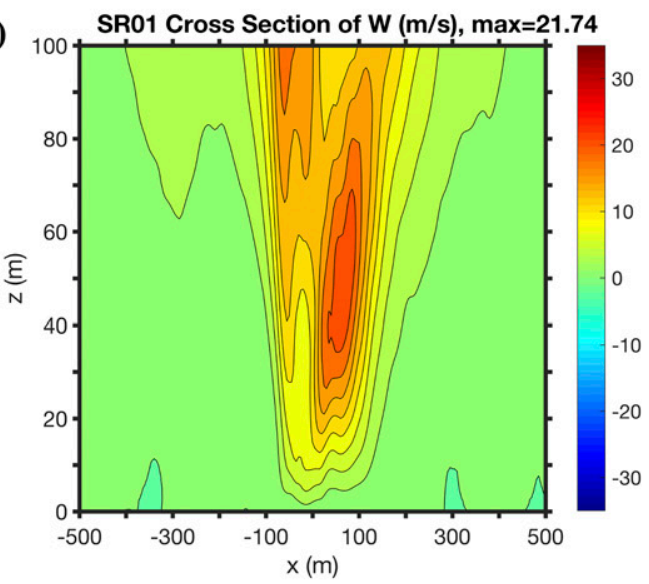

(f)

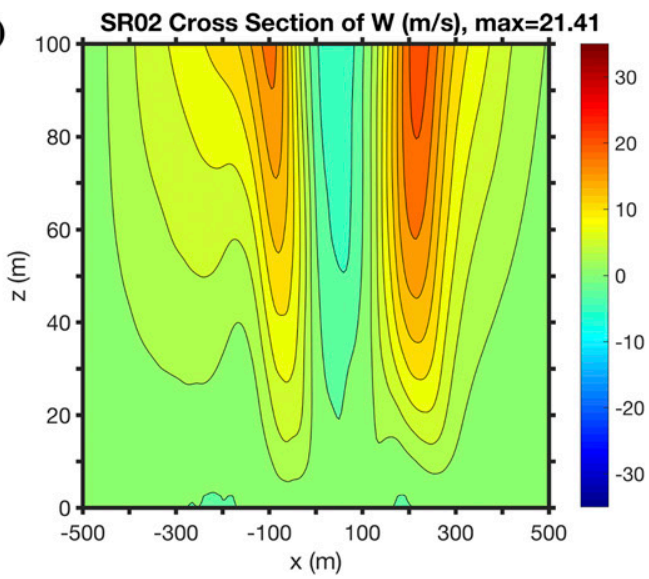

FIG. 17. Zonal, time-mean, vertical cross sections of (left) horizontal wind speed $S$ and (right) vertical wind speed $W$ through the center of the simulated (a),(b) SR005; (c),(d) SR01; and (e),(f) SR02 vortices. Note that the vertical axis is stretched by a factor of 10 .

error is the dominant contributor to total error in these cases. It is clear that radar range, taken in isolation, is a weak predictor of total error in these cases, with the interval between the $E_{\text {tot }} 5$ th and 95 th percentiles spanning nearly the entire range from 0 to 1 over most of the domain in Fig. 18. As with $E_{\mathrm{smth}}$, the distribution as a function of
$U_{\text {obs }}$ is less diffuse but also varies noticeably from simulation to simulation, whereas the distribution as a function of $\operatorname{dev}_{\text {pred }}$ is both more consistent and monotonic.

For small $\operatorname{dev}_{\text {pred }}$ (i.e., good alignment between the true wind direction and the radar beam), Fig. 18 indicates a median $E_{\text {tot }}$ of approximately 0.9 or, in other words, a 
(a)

a)

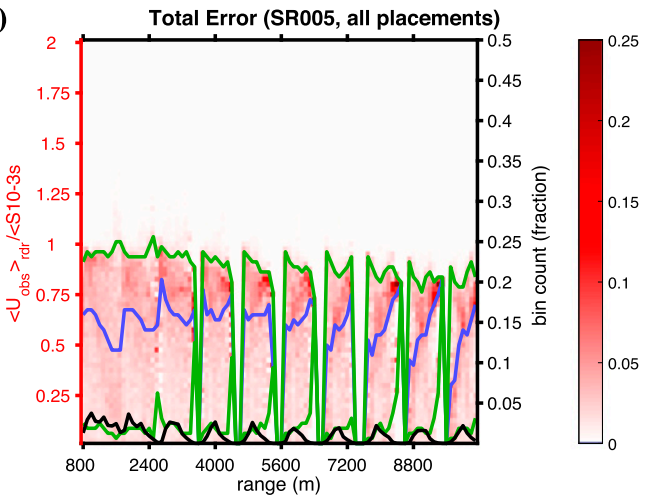

(c)

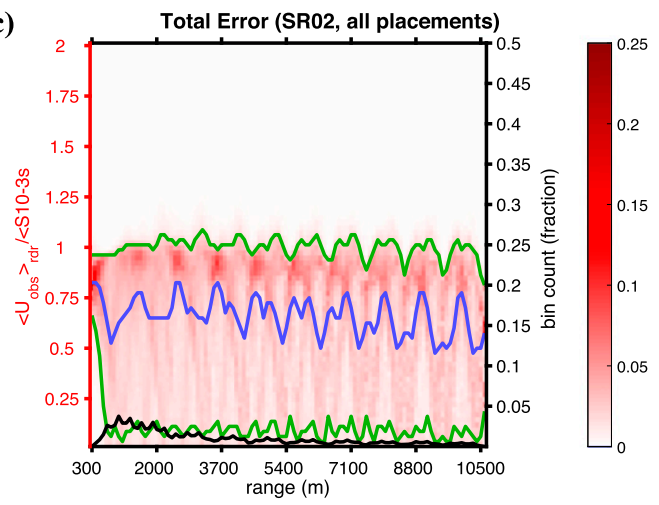

(b)

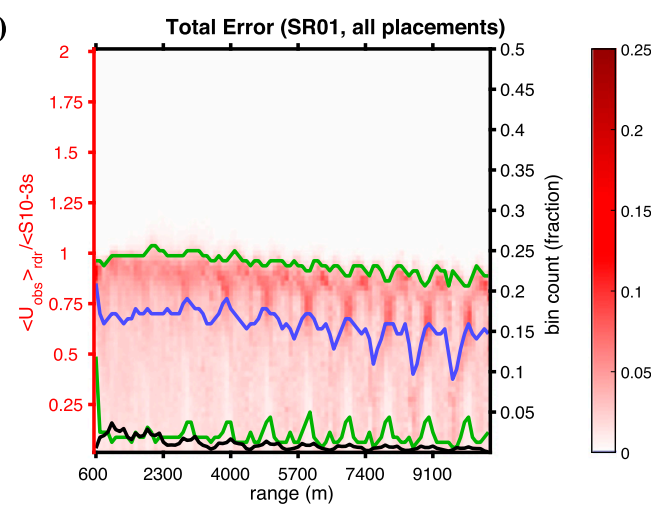

(d)

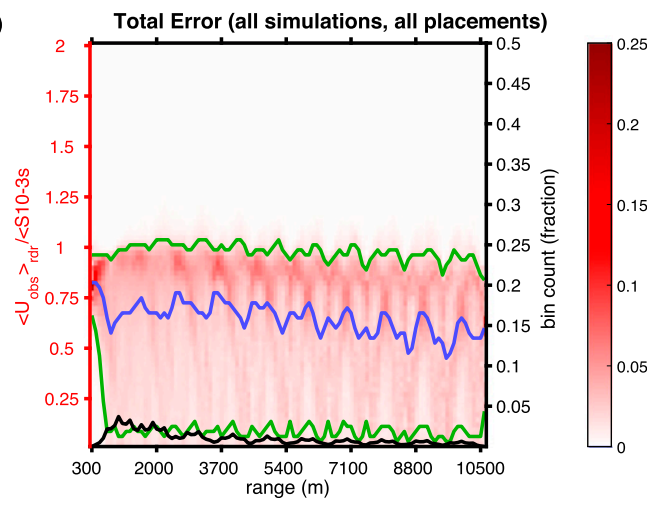

FIG. 18. As in Fig. 7, but for total error as a function of radar range.

median underestimate of S10-3s of roughly $10 \%$. This corresponds to an error of $5-10 \mathrm{~m} \mathrm{~s}^{-1}$ at most for the simulations shown here, which, again, is comparable to the uncertainty commonly specified for real radar observation ranges of strong tornadoes. Also, note that the value of 0.9 for $E_{\text {tot }}$ is an improvement over the corresponding median value of approximately 0.85 for combined $E_{\mathrm{smth}}$ and $E_{\mathrm{dir}}$, because of the countering effect of $E_{\text {elev }}$ noted in the previous section. Taken in conjunction with the general uncertainly regarding vertical wind profiles in tornadic surface layers, this result suggests that performing a height correction of tornadic wind radar observations in an effort to remove elevation error may not be advisable in cases where the target altitude is included in the resolution volume since that may actually increase the total error.

However, the underestimate increases markedly and becomes increasingly difficult to predict as $\operatorname{dev}_{\text {pred }}$ increases, with estimates of $E_{\text {tot }}$ based on dev pred being just as unreliable for $\operatorname{dev}_{\text {pred }}>40^{\circ}$ (judging from the range between the 5th and 95th percentiles) as estimates based on radar range. Thus, as with $E_{\text {smth }}$ (Fig. 11), the results are range dependent even though plotting $E_{\text {tot }}$ as a function of $d_{\text {ev }}$ pred provides the clearest depiction of the statistical distribution. For example, as shown in Fig. 21, the median underestimate at small $\operatorname{dev}_{\text {pred }}$ increases from roughly $10 \%$ for the $1 \mathrm{~km}$ south radar placement to $20 \%-25 \%$ for the $10 \mathrm{~km}$ south radar placement. Again, note that this increase is slightly less than the corresponding increase in $E_{\text {smth }}$ shown in Fig. 11 caused by the counteracting effect of $E_{\text {elev }}$.

\section{e. Estimates of global maximum S10-3s}

The foregoing analysis seeks to account for the wind speed at every location within the vortex. However, in light of the current manner in which tornadoes are rated, it is relevant to focus specifically on how well the maximum wind speeds within the vortex are estimated by radar observations. Figures 22-24 show the distributions of the maximum radar-estimated 3-s gust [i.e. $\left\langle U_{\mathrm{obs}}\right\rangle_{\mathrm{rad}}$ in (8a)] and the true maximum S10-3s valid at each scan time during the observation period ( 150 scans in all) for each simulation, plotted as functions of radar placement.

Except for the radar placement $1 \mathrm{~km}$ south of the SR01 vortex track, the global maximum S10-3s (thick, solid, black lines) is always underestimated by the global maximum $\left\langle U_{\text {obs }}\right\rangle_{\text {rad }}$ (thick, solid red lines) by $15 \mathrm{~m} \mathrm{~s}^{-1}$ or more. Moreover, the underestimate worsens with increased radar distance, particularly for SR01. With the radar placed $10 \mathrm{~km}$ south of the track, the low-swirl 
(a)

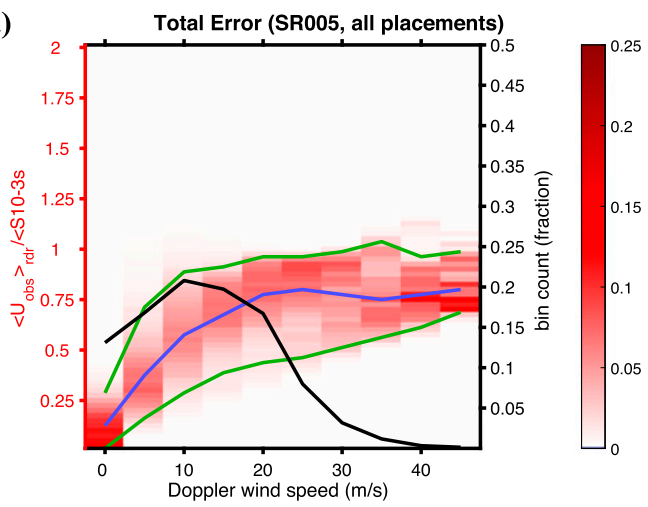

(c)

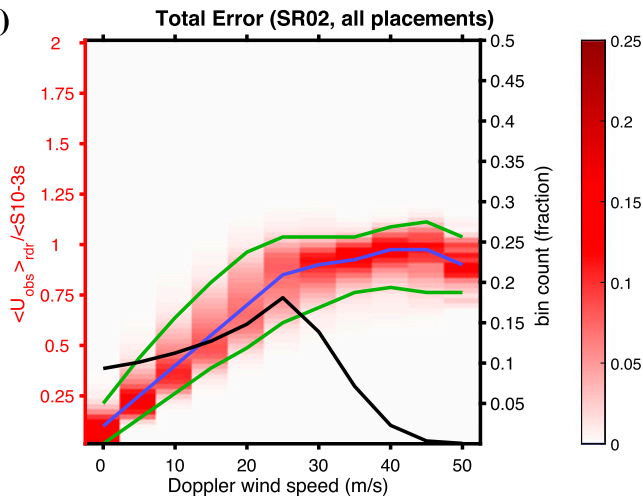

(b)

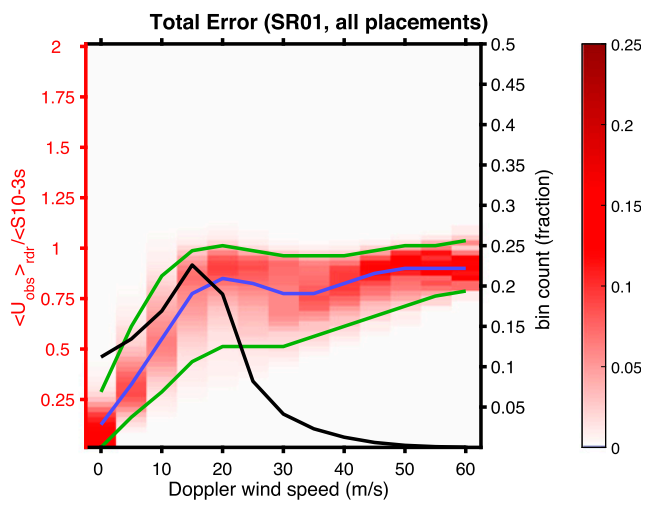

(d)

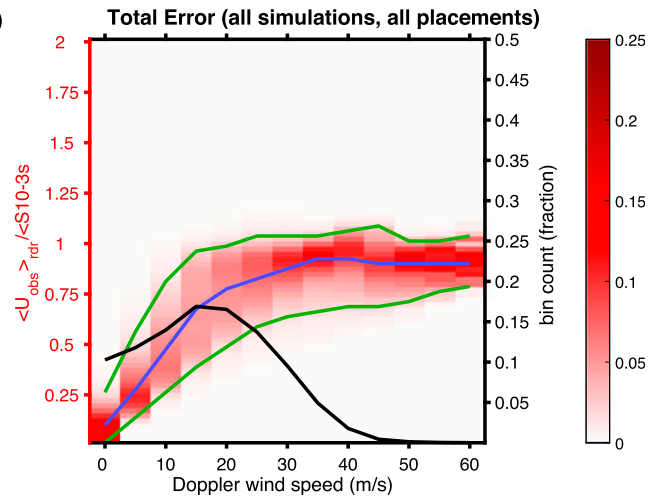

FIG. 19. As in Fig. 18, but plotted as a function of observed Doppler wind speed.

vortex would be rated EF0 based on radar measurements, the medium-swirl vortex would be rated EF1, and the high-swirl vortex would be rated EF2; however, in reality, the medium-swirl vortex is the most violent of the three and each vortex has a true maximum S10-3s exceeding $75 \mathrm{~m} \mathrm{~s}^{-1}$ [the threshold for an EF4 rating as given in Wind Science and Engineering Center (2006); see also Table 1]. With regard to intensity fluctuations (indicated by the range between the 5th and 95th percentiles, marked by the short dashed lines), the results appear to vary with vortex type, with the variability of the radar measurements agreeing well with the actual variability of the low-swirl vortex (Fig. 19) but giving a poor representation, particularly at longer range, for the high-swirl vortex.

However, the global maximum $\left\langle U_{\text {obs }}\right\rangle_{\text {rad }}$ provides a surprisingly good estimate for the median maximum S10-3s much of the time. In fact, if we take the median maximum S10-3s as a measure of the "time mean" intensity of the vortex, similar to Dahl et al. (2017), the estimation error is $<10 \mathrm{~m} \mathrm{~s}^{-1}$ for radar placements out to $5 \mathrm{~km}$ for the low-swirl vortex, $3 \mathrm{~km}$ for the mediumswirl vortex, and $7 \mathrm{~km}$ for the high-swirl vortex. It must be acknowledged that this result is influenced somewhat by the stabilizing effect of the constant background conditions (e.g., ambient angular momentum and vertical forcing) on the time-mean state of the vortex; in reality, those conditions can vary rapidly in time, in which case a 5-min observation period may be too long to consider the time-mean state of the vortex as representative of a steady state. To evaluate the impact these artificial constraints may have on the analysis, the model output and radar observations for each simulation were divided into time segments ranging from 10 to $150 \mathrm{~s}$; the true time-mean maximum S10-3s and the global maximum radar estimate were calculated for each segment and the results were averaged over the full 300-s period.

The results are shown in Fig. 25; reducing the "steady state" window from 300 to $60 \mathrm{~s}$ reduced the global radarestimated maximum by roughly $5 \mathrm{~m} \mathrm{~s}^{-1}$ on average for closer placements. (The result for placements farther away is less sensitive, since the local fluctuations that produce the variation in global maxima from segment to segment are largely smoothed out.) Reviewing Figs. 22-24, this reduction actually improves the estimate from the $1 \mathrm{~km}$ south placement for the medium-swirl and high-swirl vortices (since using the full 300-s period produces an overestimate of the true time-mean maximum), but its general effect elsewhere is to make the estimate worse. Even when the steady-state assumption 
(a)

a)

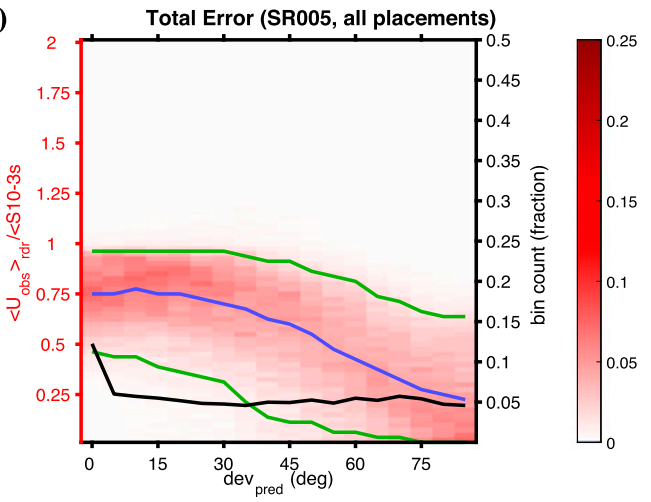

(c)

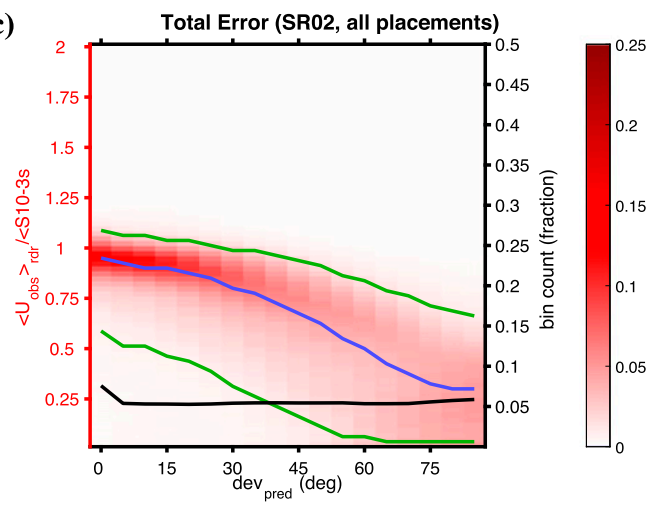

(b)

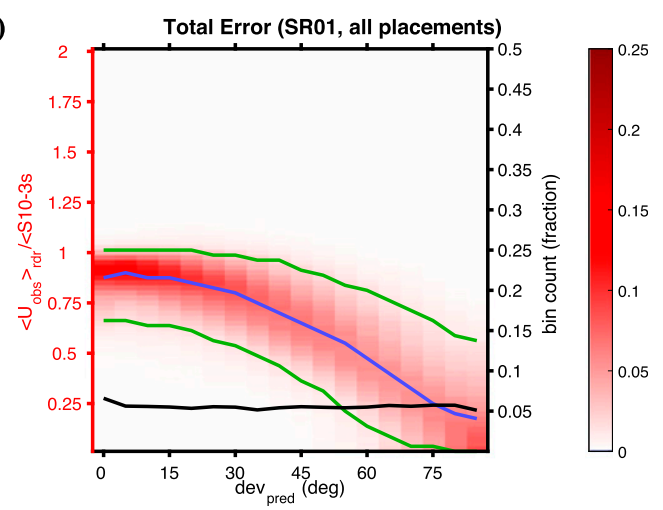

(d)

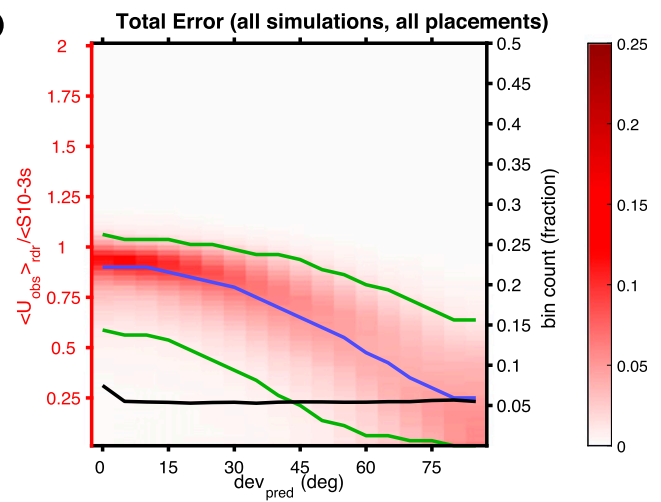

FIG. 20. As in Fig. 18, but plotted as a function of predicted deviation from beam.

is applied for only $1 \mathrm{~min}$, though, the average estimate of the time-mean maximum based on the observed global maximum is within $10 \mathrm{~m} \mathrm{~s}^{-1}$ for radar placements out to $2 \mathrm{~km}$ for the low-swirl and medium-swirl vortices and $5 \mathrm{~km}$ for the high-swirl vortex. This is comparable to the expected result of placing one perfect in situ sensor in the SR02 vortex path, two perfect sensors in the SR01 vortex path, or five perfect sensors in the SR005 vortex path as reported by Dahl et al. (2017). However, the perfect sensor results are averaged over thousands of samples, and the outcome in any individual case (i.e., a specific set of perfect sensors being placed in the path of a specific tornado) is uncertain. Contrasting this with the current experiments, in which placing a single radar near the vortex track is sufficient to obtain a comparable estimate (without the need to average over thousands of cases), underscores the potential value of rapid scan mobile radar measurements of tornadoes, notwithstanding the error sources examined here.

\section{Summary and conclusions}

This study provides quantitative insight regarding rapid scan mobile Doppler radar observation errors of tornadic wind fields, specifically with respect to estimates of 3-s gusts at 10-m height (S10-3s) relating to intensity ratings on the enhanced Fujita (EF) scale. High-resolution simulations of low-swirl, medium-swirl, and high-swirl tornado-like vortices were observed from positions ranging from 1 to $10 \mathrm{~km}$ south of their respective tracks using an emulated RaXPol radar. The resulting time-averaged measurements were then compared to the maximum "true" S10-3s corresponding to each observation location within the vortex at each scan time. In keeping with the current practice in which a tornado is rated based on its global maximum intensity, we also compared the true and radar-estimated S10-3s maxima for each radar scan as well as the true and radarestimated global S10-3s maxima over each vortex track.

The total error was partitioned into "smoothing," "direction," "elevation," "temporal sampling interval," and "representativeness" error sources, each of which was analyzed as a function of radar range, observed Doppler wind speed, and predicted deviation between the radar beam and the wind direction (based on a smoothed wind field assumed to be axisymmetric), or "dev pred." For these cases, temporal sampling and representativeness errors were found to be largely negligible when averaging over the prescribed 3-s period. It should be borne in mind, however, that these results 
(a)

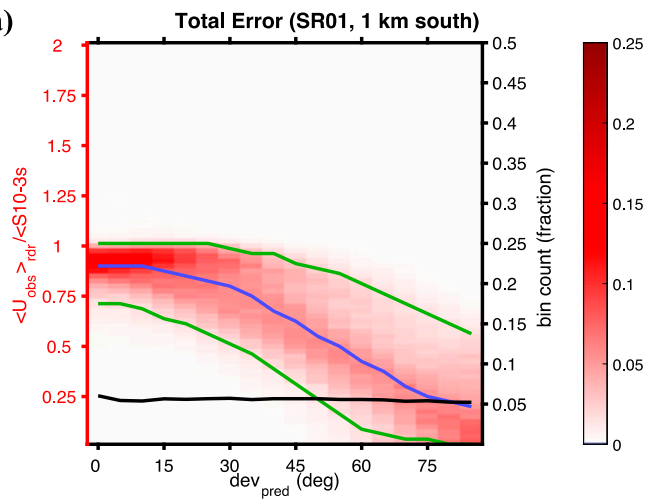

(b)

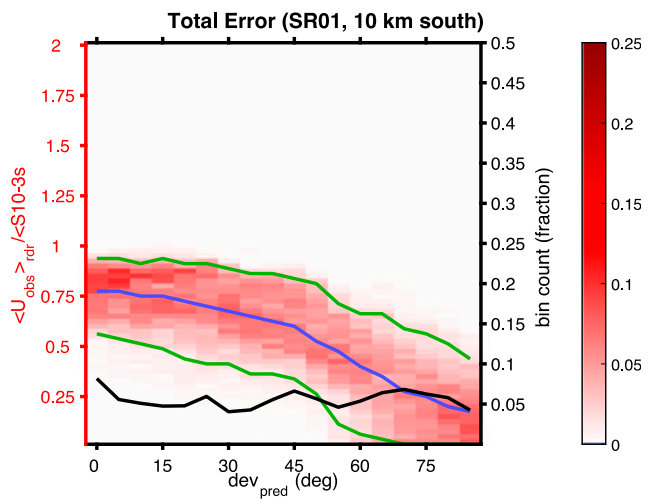

Fig. 21. As in Fig. 20, but only for simulation SR01 for radar placements (a) 1 and (b) $10 \mathrm{~km}$ south of the vortex track.

are based on a 2-s scan period. For mobile Doppler radars with substantially longer scan periods, it is impossible to obtain a time average that approximates the desired gust duration of $3 \mathrm{~s}$. Instead, estimates of S10-3s from those data must rely solely on instantaneous measurements; as shown in Dahl et al. (2017) (e.g., see their Fig. 4), discrepancies between the instantaneous $10-\mathrm{m}$ wind and the local 10-m, 3-s gust may be massive, potentially in excess of $50 \%$ for small, rapidly translating, intense subvortices. Therefore, continued effort to improve mobile radar scan rates seems warranted.

As expected, elevation error was generally found to contribute to overestimation and vary as a function of range, while direction error was generally found to contribute to underestimation and vary most consistently with $\operatorname{dev}_{\text {pred }}$. However, despite the prior expectation that smoothing error would be mostly tied to the resolution volume expanding with increased distance from the radar, we instead found that smoothing error was most closely tied to dev $\mathrm{pred}_{\text {pin }}$ these cases (caused by the strong gradients in observed Doppler velocity in regions of a vortex where the deviation from the radar beam is large), although substantial dependence on radar range was observed as well. The results with respect to observed Doppler wind speed generally mimicked the results with respect to $\operatorname{dev}_{\text {pred, }}$, suggesting that winds within the vortex that were largely aligned with the radar beam were observed equally well regardless of wind speed. When combined, the median net effect of the error sources was found to be an underestimate of S10-3s of approximately $10 \%$ for closer-range observations with small $\mathrm{dev}_{\text {pred }}$. Shifting the radar placement from $1 \mathrm{~km}$ to $10 \mathrm{~km}$ south of the track increased the underestimate by $\sim 10 \%$ on average, while an increase in $\operatorname{dev}_{\text {pred }}$ corresponded to more pronounced increases in both the median magnitude and the uncertainty of the underestimate.
In contrast, the errors in estimating the maximum S10-3s valid for a given radar scan time, as well as the errors in estimating the global maximum S10-3s, showed a distinct dependence on radar range. While the estimated global maximum from the closest radar placement ( $1 \mathrm{~km}$ south of the track) was within $10 \mathrm{~m} \mathrm{~s}^{-1}$ of the true global maximum for the medium-swirl vortex, all other placements produced underestimates of at least $15 \mathrm{~m} \mathrm{~s}^{-1}$ and the $10-\mathrm{km}$ placements produced underestimates of $30-40 \mathrm{~m} \mathrm{~s}^{-1}$, amounting to an EF rating two to four categories below what the actual global S10-3s maxima would justify. On the other hand, the median true maximum $\mathrm{S} 10-3 \mathrm{~s}$ (which we consider as a measure of the "time-mean maximum intensity") was estimated to within $10 \mathrm{~m} \mathrm{~s}^{-1}$ by the closer radar placements for all three simulated vortices, suggesting that consistently obtaining a good estimate of the time-mean

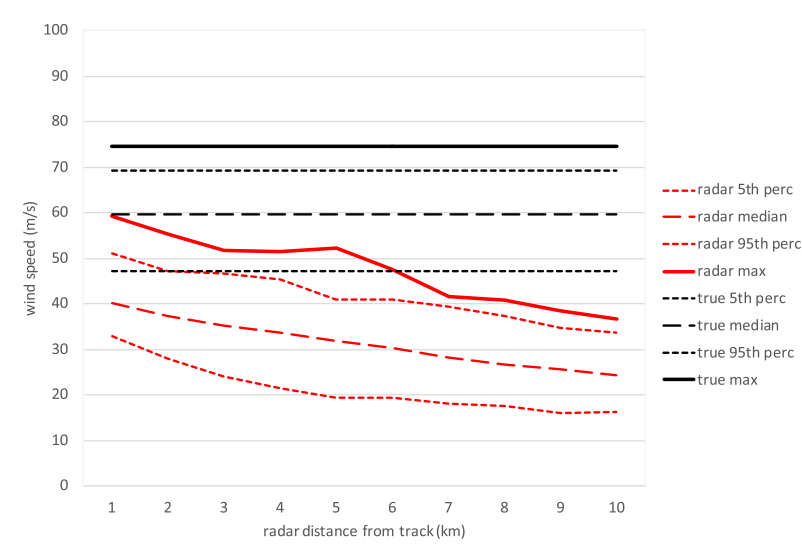

FIG. 22. Median (long dash), 5th and 95th percentiles (short dash), and maximum values (thick solid) of radar-estimated (red) and true (black) maximum 10-m, 3-s gust speeds $\left(\mathrm{m} \mathrm{s}^{-1}\right)$ valid at radar scan times during the observation period $(n=150)$ for simulation SR005, plotted as a function of minimum radar distance from the vortex track $(\mathrm{km})$. 


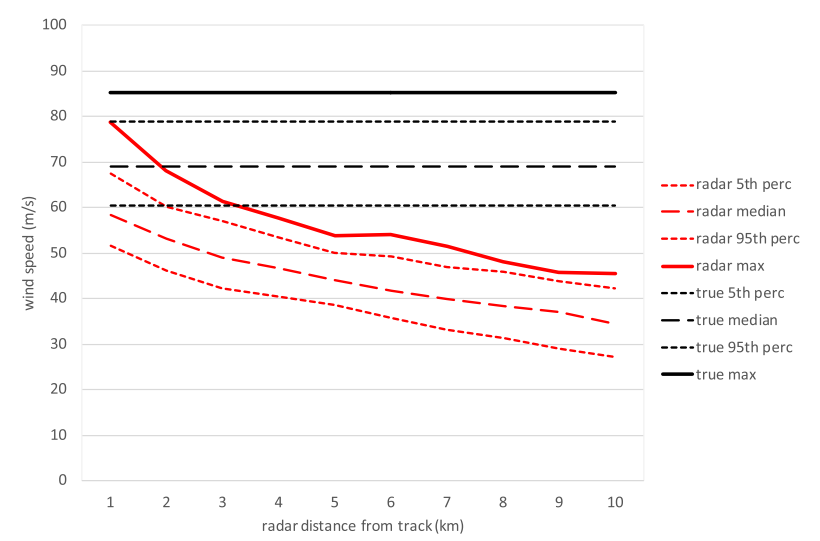

FIG. 23. As in Fig. 22, but for simulation SR01.

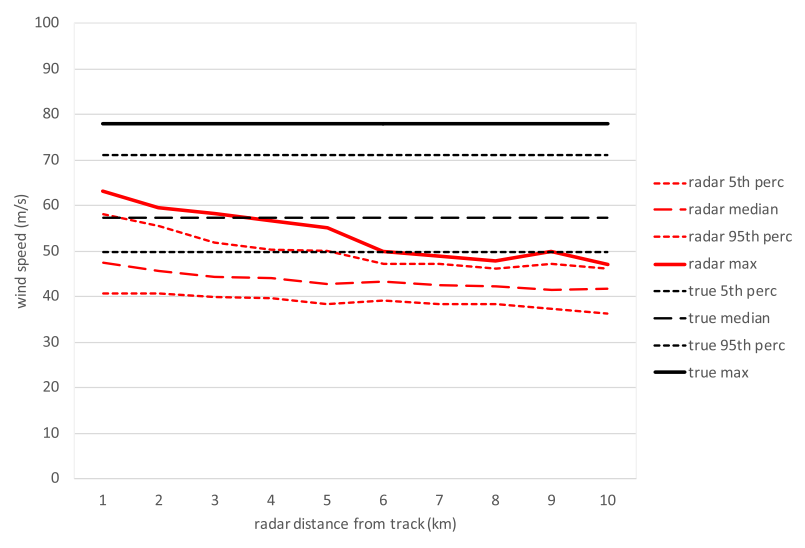

FIG. 24. As in Fig. 23, but for simulation SR02. maximum intensity (rather than the EF scale rating) of a tornado from close-range mobile Doppler observations may be possible.

Since this study is intended mainly as an introduction and illustration of concepts, these data have obvious limitations. For example, only three vortices are examined, all of similar intensity and translating at the same (constant) speed over level terrain, which is not representative of the wide variety of scenarios in which actual mobile Doppler measurements of tornadoes occur. In a similar vein, only a limited range of radar placements was tested here, all of them directly south of the midpoint of the vortex track. While this was done in an effort to provide a "best case" scenario for a stationary radar, based on the prior assumption that the best results would come from having the radar perpendicular to the midpoint of the track with a substantial observation period on either side of the point of closest approach, the results make it clear that a best case scenario is not simply a function of range. Because of the impacts of the deviation from beam, placing the radar directly ahead of (or, in the interests of safety, behind) the tornado, even at longer range, may produce better estimates of the maximum wind speed than placing the radar beside the track would, even if the former placement is farther away.

We also note in passing that the most general way to mitigate problems associated with wind direction versus beam direction would be to employ an additional radar and perform dual-Doppler analysis. However, the task of positioning two mobile Doppler radars to make concerted, finescale scans of a tornado is daunting, with only a handful of successful deployments reported in the literature (e.g., Wurman et al. 2010; Atkins et al. 2012; Wakimoto et al. 2016). Also, the best procedure for mapping tornadic wind data from nonsimultaneous scans to a common grid and analysis time is unclear. Shapiro et al. (2015) report superior results for trajectory analysis of a simulated tornadic supercell using advection correction based on the method of Gal-Chen (1982), compared with results from linear interpolation or mean-wind advection. However, the potential for aliasing errors noted for the former method could theoretically be significant for intense, rapidly evolving features (e.g., subvortices) within a tornado, and this possibility has not yet been tested. Finally, obtaining volume scans sufficient for dual-Doppler analysis currently requires a time period substantially longer than $3 \mathrm{~s}$; the best case we can envision using current radar capabilities would be two RSDOWs (Wurman and Randall 2001) taking synchronized volume scans, which would still require a minimum of $7 \mathrm{~s}$ to complete and, therefore, would be vulnerable to the same errors in estimating S10-3s from instantaneous data that were discussed previously. Therefore, we believe that focusing on single-Doppler measurements is likely to be more relevant to the evaluation of S10-3s from real tornado data in the near future.

Finally, the simplifications employed here have their own repercussions. For example, the simple geometrical treatment for dev pred shown here produces errors that may then increase the uncertainty in the error estimates that use $\operatorname{dev}_{\text {pred }}$ as a basis; employing a more sophisticated analysis like GBVTD instead could reduce such contamination. Also, because of resolution constraints and the "clear air" nature of the vortex simulations, errors arising from statistical processing, nonuniform scattering, and deviant motion of scatterers are not examined here. Applying the same method to simulations that include lofted debris (e.g., described by Cheong et al. 2017) and including spectral and dual-polarization products in the radar emulation would enable quantification of those errors as well. Finally, the power distribution defined in (5) neglects any contamination from sidelobes and we do not attempt to simulate any 


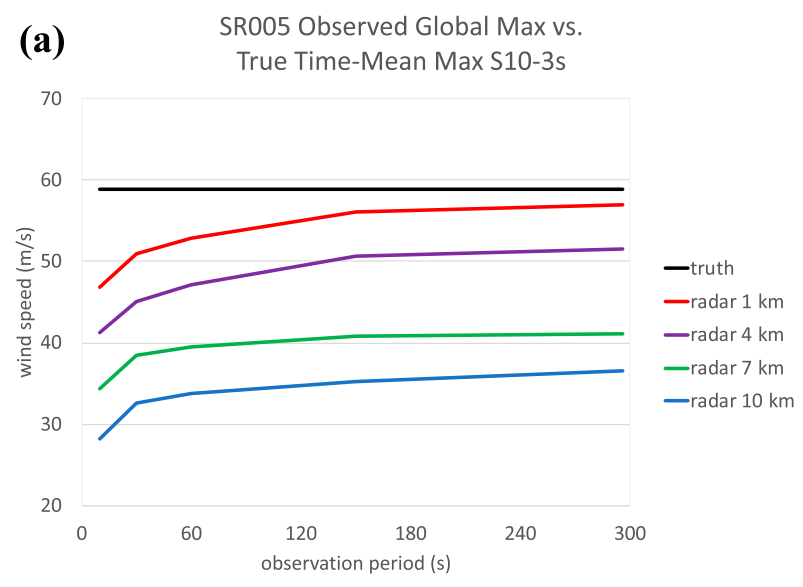

(b) SR01 Observed Global Max vs.

True Time-Mean Max S10-3s

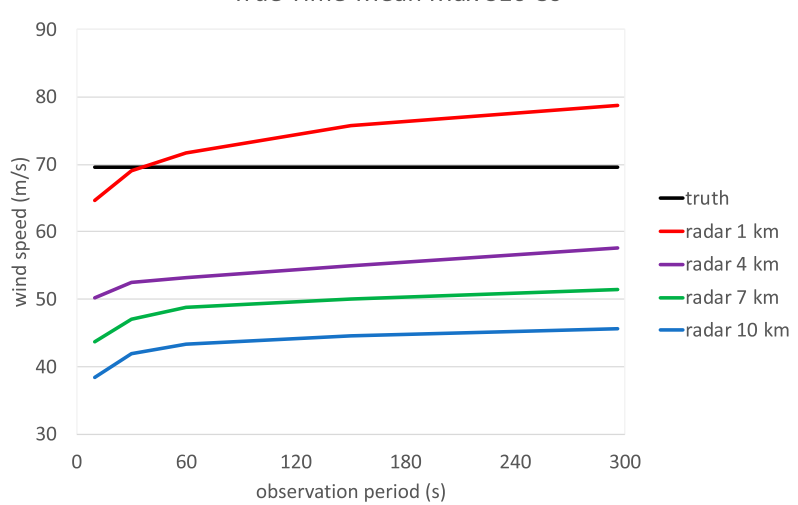

(c) SR02 Observed Global Max vs. True Time-Mean Max S10-3s

80

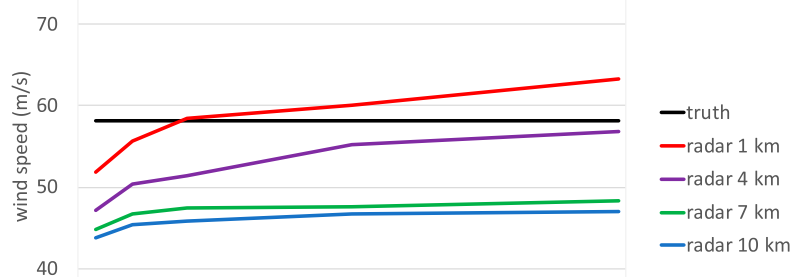

40

30

0

60

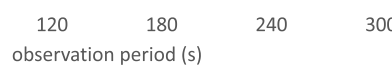

FIG. 25. True time-mean maximum 10-m, 3-s gust (black) and mean global maximum radar-estimated $10-\mathrm{m}, 3$-s gusts from $1 \mathrm{~km}$ south (red), $4 \mathrm{~km}$ south (purple), $7 \mathrm{~km}$ south (green), and $10 \mathrm{~km}$ south (blue) radar placements, plotted with respect to the duration of the period of observation for simulations (a) SR005, (b) SR01, and (c) SR02.

interaction of the beam with the surface, which may also be significant in real observations. A comprehensive effort at quantification of errors would need to test all of these variables rigorously. However, we believe that efforts such as these are reasonable and worthwhile steps to take, particularly in light of the continuing challenges related to improved understanding and categorization of tornadoes.

Acknowledgments. This work was supported in part by the National Science Foundation through Grant AGS1265899. The simulations analyzed here were produced in collaboration with George Bryan and Richard Rotunno. We acknowledge high-performance computing support from Yellowstone (ark:/85065/d7wd3xhc) provided by the Computational and Informational Systems Laboratory at the National Center for Atmospheric Research (NCAR), which is also sponsored by the National Science Foundation. We also thank the anonymous reviewers for their assistance in refining this manuscript.

\section{APPENDIX}

\section{Simple Prediction of Wind Direction within a Vortex}

Sophisticated methods have been developed for retrieving the structure of a vortex from single-Doppler velocity data, such as the commonly used GBVTD technique (Lee et al. 1999). However, merely a reasonable approximation of the true wind direction will suffice for our work, and, therefore, a more streamlined approach is employed here. Before the analysis, a $200 \mathrm{~m} \times$ $200 \mathrm{~m}$ top-hat filter is applied to the radar observations to reduce possible contamination by subvortices. The resulting filtered Doppler wind field is assumed to be roughly axisymmetric, possibly consisting of both tangential and radial components, with the inflow angle $\alpha$ (i.e., the angle between the true wind direction and the direction tangent to the vortex) depending only on the distance from the vortex center. Under these assumptions, $\alpha$ (and, subsequently, the expected wind direction) can be obtained at any location within the vortex.

First, the location of the vortex center must be estimated in a manner that accounts for inflow and the effects of vortex size and distance from the radar [described, e.g., in Wood and Brown (1992)]. As shown in Fig. A1, inflow and radar beam direction variations across the vortex influence the difference $\delta$ between the (unknown) true center location $\mathbf{C}\left(=x_{C} \hat{\mathbf{i}}+y_{C} \hat{\mathbf{j}}\right)$ and the initial estimate $\mathbf{C}^{*}$ defined (e.g., as in Wurman et al. 2013) as the midpoint between the locations of the maximum and minimum Doppler velocities, where the wind direction is assumed to be parallel to the radar beam. We denote these locations as $\mathbf{R}_{\max }=x_{\max } \hat{\mathbf{i}}+y_{\max } \hat{\mathbf{j}}$ and $\mathbf{R}_{\min }=x_{\min } \hat{\mathbf{i}}+y_{\min } \hat{\mathbf{j}}$, respectively. (Note that all $x$ and $y$ distances are relative to the location of the radar, and that the vortex is assumed to rotate counterclockwise.) In other words, 


$$
\mathbf{C}^{*}=0.5\left(x_{\max }+x_{\min }\right) \hat{\mathbf{i}}+0.5\left(y_{\max }+y_{\min }\right) \hat{\mathbf{j}}
$$

From Fig. A1, applying the convention that positive azimuth is measured counterclockwise from true north, the radar-relative azimuth angles to $\mathbf{R}_{\max }$ and $\mathbf{R}_{\min }$ (denoted $\theta_{\max }$ and $\theta_{\min }$ ) are related to the corresponding (unknown) vortexcenter-relative azimuth angles $\left(\phi_{\max }\right.$ and $\left.\phi_{\min }\right)$ as follows:

$$
\begin{aligned}
& \theta_{\text {max }}=\phi_{\text {max }}+\alpha-\frac{\pi}{2}, \\
& \theta_{\text {min }}=\phi_{\text {min }}+\alpha+\frac{\pi}{2} .
\end{aligned}
$$

(Note $\alpha$ is defined as negative for inflow and positive for outflow.) Subtracting (A3) from (A2) and using the fact that $\phi^{*}=2 \pi-\left(\phi_{\max }-\phi_{\min }\right)$ yields

$$
\phi^{*}=\pi-\left(\theta_{\max }-\theta_{\min }\right) .
$$

Since both $\mathbf{C}$ and $\mathbf{C}^{*}$ are equidistant from $\mathbf{R}_{\max }$ and $\mathbf{R}_{\min }$, we know that the line from $\mathbf{C}^{*}$ to $\mathbf{C}$ bisects $\phi^{*}$ and is perpendicular to and bisects the line from $\mathbf{R}_{\max }$ to $\mathbf{R}_{\min }$. Thus,

$$
\begin{aligned}
\tan \left(\frac{\phi^{*}}{2}\right) & =\frac{0.5\left|\mathbf{R}_{\max }-\mathbf{R}_{\min }\right|}{\delta} \rightarrow \\
\delta & =\frac{\left|\mathbf{R}_{\max }-\mathbf{R}_{\min }\right|}{2 \tan \left(\frac{\phi^{*}}{2}\right)} .
\end{aligned}
$$

The unit vector in the direction from $\mathbf{C}^{*}$ to $\mathbf{C}$ is given by $\hat{\mathbf{s}}=\frac{-\left(\mathbf{R}_{\max }-\mathbf{R}_{\min }\right) \times \hat{\mathbf{k}}}{\left|\mathbf{R}_{\max }-\mathbf{R}_{\text {min }}\right|}=\frac{\left(y_{\text {min }}-y_{\text {max }}\right) \hat{\mathbf{i}}-\left(x_{\min }-x_{\max }\right) \hat{\mathbf{j}}}{\left|\mathbf{R}_{\max }-\mathbf{R}_{\min }\right|}$.

Therefore, the location of the vortex center is

$$
\begin{aligned}
\mathbf{C}=\mathbf{C}^{*}+\delta \hat{\mathbf{s}}= & \mathbf{C}^{*}+\frac{1}{2 \tan \left(\frac{\phi^{*}}{2}\right)} \\
& \times\left[\left(y_{\text {min }}-y_{\text {max }}\right) \hat{\mathbf{i}}-\left(x_{\text {min }}-x_{\text {max }}\right) \hat{\mathbf{j}}\right],
\end{aligned}
$$

where $\mathbf{C}^{*}$ is obtained from (A1) using the known $x_{\min }$, $x_{\max }, y_{\min }$, and $y_{\max }$ and $\phi^{*}$ is obtained from (A4) using the known $\theta_{\min }$ and $\theta_{\max }$.

Having obtained $\mathbf{C}$, the inflow angle is now estimated as a function of distance from the vortex center by interpolating the Doppler velocity field to a circle surrounding $\mathbf{C}$ at a given radius and determining the radarrelative locations $\mathbf{R}_{\text {max }_{\text {int }}}(r)$ and $\mathbf{R}_{\text {min }_{\text {int }}}(r)$ and radar-relative azimuth angles $\theta_{\text {max }_{\text {int }}}(r)$ and $\theta_{\min _{\text {int }}}(r)$ of the locations of maximum and minimum Doppler velocity (respectively) on the circle. At the location of the Doppler velocity maximum, the unit vector along the radar beam (again,

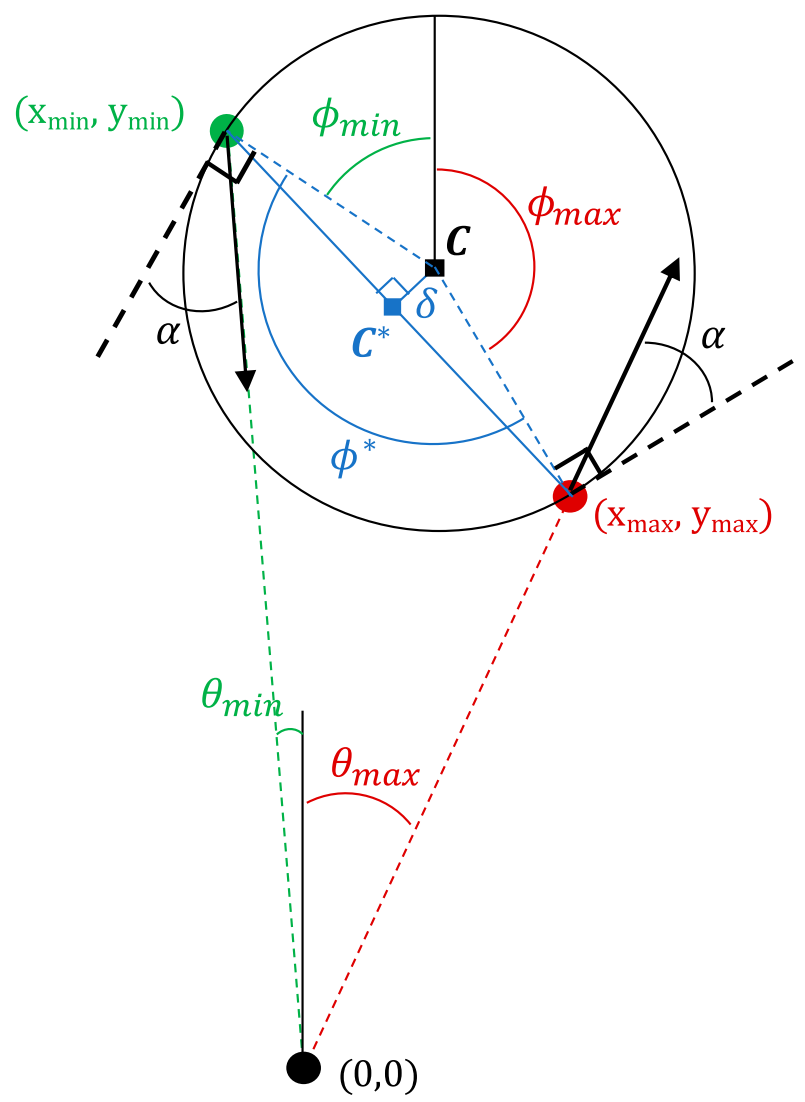

FIG. A1. Geometric analysis of a convergent, axisymmetric, cyclonic vortex (black circle) observed by a single Doppler radar (black dot), based on the locations of maximum outbound (red dot) and inbound (green dot) velocity. Additional symbols and terms are described in the text.

assumed parallel to the true wind when the magnitude of the Doppler velocity is maximized) is

$$
\widehat{\mathbf{s}_{1}}(r)=\sin \left[\theta_{\max _{\mathrm{int}}}(r)\right] \hat{\mathbf{i}}+\cos \left[\theta_{\max _{\mathrm{int}}}(r)\right] \hat{\mathbf{j}} .
$$

The unit vector tangent to the vortex at this location is

$$
\widehat{\mathbf{s}_{2}}(r)=\frac{\left[\mathbf{C}-\mathbf{R}_{\text {max }_{\text {int }}}(r)\right] \times \hat{\mathbf{k}}}{\left|\mathbf{C}-\mathbf{R}_{\text {max }_{\text {int }}}(r)\right|} .
$$

The inflow angle at this location is then estimated as

$$
\alpha_{\max }(r)=\left\{\begin{array}{ll}
\sin ^{-1}\left[\left|\widehat{\mathbf{s}_{1}}(r) \times \widehat{\mathbf{s}_{2}}(r)\right|\right], & \widehat{\mathbf{s}_{1}}(r) \cdot \widehat{\mathbf{s}_{2}}(r) \geq 0 \\
\sin ^{-1}\left[\left|\widehat{\mathbf{s}_{2}}(r) \times \widehat{\mathbf{s}_{1}}(r)\right|\right], & \widehat{\mathbf{s}_{1}}(r) \cdot \widehat{\mathbf{s}_{2}}(r)<0
\end{array} .\right.
$$

Again, note that negative $\alpha$ indicates inflow. The calculation is repeated for the location of the Doppler velocity minimum by replacing $\theta_{\text {max }_{\text {int }}}(r)$ with $\theta_{\text {min }_{\text {int }}}(r)$ in (A8), $\mathbf{R}_{\text {max }_{\text {int }}}(r)$ with $\mathbf{R}_{\text {min }_{\text {int }}}(r)$ in (A9), and $\alpha_{\max }(r)$ with $\alpha_{\min }(r)$ 
(a)

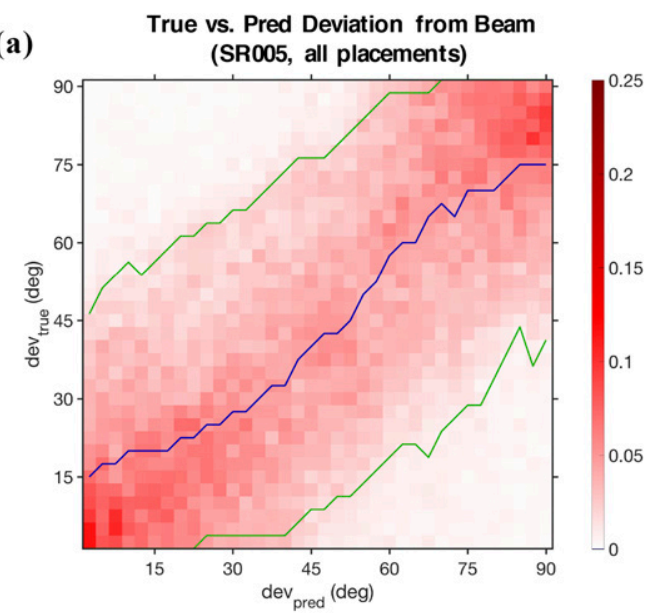

(c)

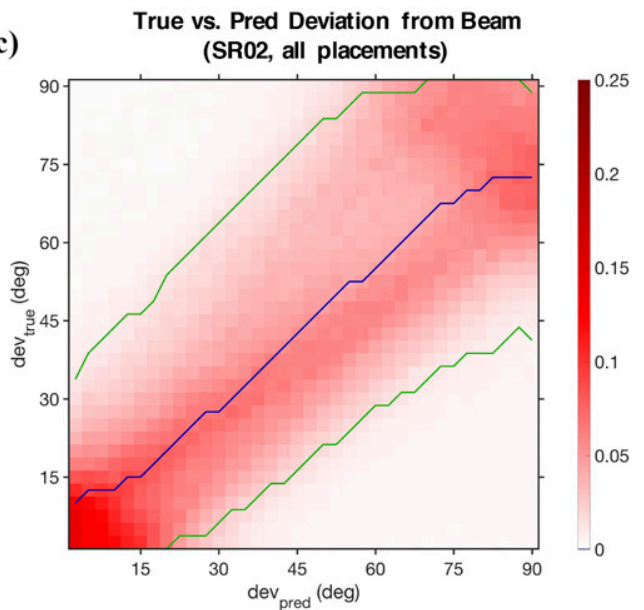

(b)
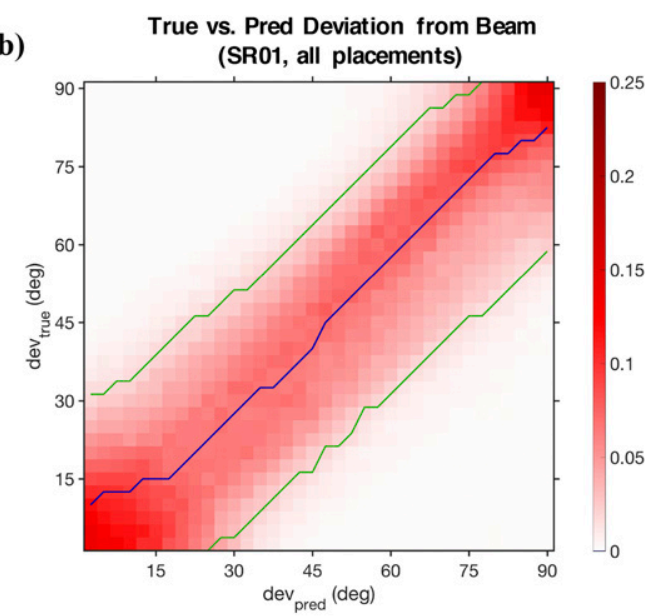

(d)

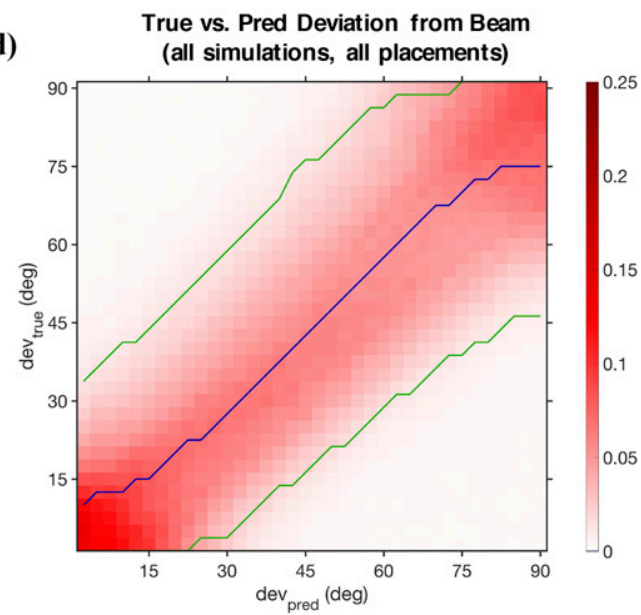

FIG. A2. True wind direction deviation from the radar beam ( $\left.\operatorname{dev}_{\text {true }}\right)$ as a function of predicted deviation ( $\left.\operatorname{dev}_{\text {pred }}\right)$, binned in $5^{\circ}$ increments, for simulation (a) SR005, (b) SR01, (c) SR02, and (d) all simulations. Median values for each $\operatorname{dev}_{\text {pred }}$ bin are plotted in blue, and 5th and 95th percentiles are plotted in green.

in (A10). The term $\alpha(r)$ is then estimated as the average of $\alpha_{\max }(r)$ and $\alpha_{\min }(r)$. This operation is performed over a range of vortex-relative radii at regular, small increments to obtain a radial profile of the inflow angle. With this profile, we can assign an estimated inflow angle to each radar observation point (i.e., the center of each resolution volume), $\mathbf{R}_{\mathrm{ob}}$, by calculating the distance from that point to the vortex center and then interpolating $\alpha(r)$ :

$$
\alpha^{*}=\alpha\left(\left|\mathbf{R}_{\mathrm{ob}}-\mathbf{C}\right|\right) .
$$

The unit vector tangent to the vortex at the observation location is

$$
\widehat{\mathbf{s}_{3}}\left(\mathbf{R}_{\mathrm{ob}}\right)=\frac{\left[\mathbf{C}-\mathbf{R}_{\mathrm{ob}}\right] \times \hat{\mathbf{k}}}{\left|\mathbf{C}-\mathbf{R}_{\mathrm{ob}}\right|} \equiv x_{3} \hat{\mathbf{i}}+y_{3} \hat{\mathbf{j}}
$$

After adding the inflow angle, the unit vector along the expected wind direction is

$$
\begin{aligned}
\widehat{\mathbf{s}_{4}}(r)= & {\left[x_{3} \cos \left(\alpha^{*}\right)+y_{3} \sin \left(\alpha^{*}\right)\right] \hat{\mathbf{i}} } \\
& +\left[y_{3} \cos \left(\alpha^{*}\right)-x_{3} \sin \left(\alpha^{*}\right)\right] \hat{\mathbf{j}} .
\end{aligned}
$$

The unit vector along the radar beam at the observation location is

$$
\widehat{\mathbf{s}_{5}}\left(\mathbf{R}_{\mathrm{ob}}\right)=\sin \left(\theta_{\mathrm{ob}}\right) \hat{\mathbf{i}}+\cos \left(\theta_{\mathrm{ob}}\right) \hat{\mathbf{j}} .
$$

The predicted deviation between the wind direction and the radar beam is then

$$
\operatorname{dev}_{\text {pred }}\left(\mathbf{R}_{\mathrm{ob}}\right)=\sin ^{-1}\left|\widehat{\mathbf{s}_{4}}\left(\mathbf{R}_{\mathrm{ob}}\right) \times \widehat{\mathbf{s}_{5}}\left(\mathbf{R}_{\mathrm{ob}}\right)\right| .
$$

To focus on the vortex itself, this analysis is confined to the continuous region around the vortex center in which the maximum filtered, interpolated Doppler wind speed for each $r$ is $\geq 20 \mathrm{~m} \mathrm{~s}^{-1}$. [In other words, if $U_{\max }(r)$ is the maximum smoothed, interpolated Doppler wind speed on a circle surrounding the vortex center with radius $r$, then the 
region used in the analysis is enclosed by a circle of radius $r_{20}$ around the vortex center in which $U_{\max }(r) \geq 20 \mathrm{~m} \mathrm{~s}^{-1}$ for all $r \leq r_{20}$.] To check the quality of the prediction, frequency diagrams of the results over all radar ranges for each simulation, as well as the results aggregated over all simulations, are shown in Fig. A2. While it is clear that the top-hat filter and assumption of stationary axisymmetry produce increased errors for SR005 (where smoothing degrades the estimate of the main vortex center location somewhat) and SR02 (where more prominent subvortices appear in the raw radar scans), the median "true" values agree well with the predicted values and the errors for individual measurements are generally small enough $(>70 \%$ within $15^{\circ},>90 \%$ within $30^{\circ}$ ) to justify using the predicted values as a reasonable approximation for our purposes.

\section{REFERENCES}

Atkins, N. T., A. McGee, R. Ducharme, R. M. Wakimoto, and J. Wurman, 2012: The LaGrange tornado during VORTEX2. Part II: Photogrammetric analysis of the tornado combined with dual-Doppler radar data. Mon. Wea. Rev., 140, 29392958, https://doi.org/10.1175/MWR-D-11-00285.1.

Bieringer, P., and P. S. Ray, 1996: A comparison of tornado warning lead times with and without NEXRAD Doppler radar. Wea. Forecasting, 11, 47-52, https://doi.org/10.1175/1520-0434(1996) 011<0047:ACOTWL>2.0.CO;2.

Bluestein, H. B., M. M. French, I. PopStefanija, R. T. Bluth, and J. B. Knorr, 2010: A mobile, phased-array Doppler radar for the study of severe convective storms: The MWR-05XP. Bull. Amer. Meteor. Soc., 91, 579-600, https://doi.org/10.1175/ 2009BAMS2914.1.

_, J. G. Ladue, H. Stein, D. Speheger, and W. P. Unruh, 1993: Doppler radar wind spectra of supercell tornadoes. Mon. Wea. Rev., 121, 2200-2221, https://doi.org/10.1175/1520-0493(1993) $121<2200$ :DRWSOS>2.0.CO;2.

Bodine, D. J., M. R. Kumjian, R. D. Palmer, P. L. Heinselman, and A. V. Ryzhkov, 2013: Tornado damage estimation using polarimetric radar. Wea. Forecasting, 28, 139-158, https://doi.org/ 10.1175/WAF-D-11-00158.1.

Brotzge, J., and S. Erickson, 2009: NWS tornado warnings with zero or negative lead times. Wea. Forecasting, 24, 140-154, https://doi.org/10.1175/2008WAF2007076.1.

Bryan, G. H., and J. M. Fritsch, 2002: A benchmark simulation for moist nonhydrostatic numerical models. Mon. Wea. Rev., 130, 2917-2928, https://doi.org/10.1175/1520-0493(2002)130<2917: ABSFMN $>2.0 . C O ; 2$.

—, N. A. Dahl, D. S. Nolan, and R. Rotunno, 2017: An eddy injection method for large-eddy simulations of tornado-like vortices. Mon. Wea. Rev., 145, 1937-1961, https://doi.org/ 10.1175/MWR-D-16-0339.1.

Cheong, B. L., D. J. Bodine, C. J. Fulton, S. M. Torres, T. Maruyama, and R. D. Palmer, 2017: Simradar: A polarimetric radar time-series simulator for tornadic debris studies. IEEE Trans. Geosci. Remote Sens., 55, 2858-2870, https://doi.org/10.1109/TGRS.2017.2655363.

Crum, T. D., and R. L. Alberty, 1993: The WSR-88D and the WSR88D operational support facility. Bull. Amer. Meteor. Soc., 74, 1669-1687, https://doi.org/10.1175/1520-0477(1993)074<1669: TWATWO $>2.0 . \mathrm{CO} ; 2$.
Dahl, N. A., D. S. Nolan, G. H. Bryan, and R. Rotunno, 2017: Using high-resolution simulations to quantify underestimates of tornado intensity from in situ observations. Mon. Wea. Rev., 145, 1963-1982, https://doi.org/10.1175/MWR-D-16-0346.1.

Doswell, C. A., III, and D. W. Burgess, 1988: Some issues of United States tornado climatology. Mon. Wea. Rev., 116, 495-501, https:// doi.org/10.1175/1520-0493(1988)116<0495:OSIOUS > 2.0.CO;2.

, H. E. Brooks, and N. Dotzek, 2009: On the implementation of the enhanced Fujita scale in the USA. Atmos. Res., 93, 554563, https://doi.org/10.1016/j.atmosres.2008.11.003.

Doviak, R. J., and D. S. Zrnić, 1993: Doppler Radar and Weather Observations. Academic Press, 562 pp.

Dowell, D. C., C. R. Alexander, J. M. Wurman, and L. J. Wicker, 2005: Centrifuging of hydrometeors and debris in tornadoes: Radar reflectivity patterns and wind measurement errors. Mon. Wea. Rev., 133, 1501-1524, https://doi.org/10.1175/MWR2934.1.

Edwards, R., 2003: Rating tornado damage: An exercise in subjectivity. Preprints, Symp. on the F-Scale and Severe-Weather Damage Assessment, Long Beach, CA, Amer. Meteor. Soc., P1.2, http://ams.confex.com/ams/pdfpapers/55307.pdf.

—, J. G. LaDue, J. T. Ferree, K. Scharfenberg, C. Maier, and W. L. Coulbourne, 2013: Tornado intensity estimation: Past, present, and future. Bull. Amer. Meteor. Soc., 94, 641-653, https://doi.org/10.1175/BAMS-D-11-00006.1.

Fiedler, B. H., 1994: The thermodynamic speed limit and its violation in axisymmetric numerical simulations of tornado-like vortices. Atmos.-Ocean, 32, 335-359, https://doi.org/10.1080/ 07055900.1994.9649501.

Fujita, T. T., 1971: Proposed characterization of tornadoes and hurricanes by area and intensity. SMRP Research Paper 91, University of Chicago, Chicago, IL, $42 \mathrm{pp}$. [Available from Wind Engineering Research Center, Box 41023, Lubbock, TX 79409.]

Gal-Chen, T., 1982: Errors in fixed and moving frame of references: Applications for conventional and Doppler radar analysis. J. Atmos. Sci., 39, 2279-2300, https://doi.org/ 10.1175/1520-0469(1982)039<2279:EIFAMF>2.0.CO;2.

Kosiba, K. A., and J. Wurman, 2013: The three-dimensional structure and evolution of a tornado boundary layer. Wea. Forecasting, 28, 1552-1561, https://doi.org/10.1175/WAF-D-13-00070.1.

Lee, W. C., B. J.-D. Jou, P.-L. Chang, and S.-M. Deng, 1999: Tropical cyclone kinematic structure retrieved from single-Doppler radar observations. Part I: Doppler velocity patterns and the GBVTD technique. Mon. Wea. Rev., 127, 2419-2439, https://doi.org/ 10.1175/1520-0493(1999)127<2419:TCKSRF > 2.0.CO;2.

Lewellen, D. C., W. S. Lewellen, and J. Xia, 2000: The influence of a local swirl ratio on tornado intensification near the surface. J. Atmos. Sci., 57, 527-544, https://doi.org/10.1175/15200469(2000)057<0527:TIOALS > 2.0.CO;2.

Marshall, T. P., 2002: Tornado damage survey at Moore, Oklahoma. Wea. Forecasting, 17, 582-598, https://doi.org/10.1175/ 1520-0434(2002)017<0582:TDSAMO>2.0.CO;2.

- D. Burgess, G. Garfield, J. Snyder, R. Smith, D. Speheger, and H. Bluestein, 2014: Ground-based damage survey and radar analysis of the El Reno, Oklahoma tornado. 27th Conf. on Severe Local Storms, Madison, WI, Amer. Meteor. Soc., 13.1, https:// ams.confex.com/ams/27SLS/webprogram/Paper254342.html.

NCDC, 2013: Storm Data. Vol. 55, No. 5, 683 pp.

Nolan, D. S., 2005: A new scaling for tornado-like vortices. J. Atmos. Sci., 62, 2639-2645, https://doi.org/10.1175/JAS3461.1.

- 2013: On the use of Doppler radar-derived wind fields to diagnose the secondary circulations of tornadoes. J. Atmos. Sci., 70, 1160-1171, https://doi.org/10.1175/JAS-D-12-0200.1. 
— N. A. Dahl, G. H. Bryan, and R. Rotunno, 2017: Tornado vortex structure, intensity, and surface wind gusts in largeeddy simulations with fully developed turbulence. J. Atmos. Sci., 74, 1573-1597, https://doi.org/10.1175/JAS-D-16-0258.1.

Pazmany, A. L., J. B. Mead, H. B. Bluestein, J. C. Snyder, and J. B. Houser, 2013: A mobile rapid-scanning X-band polarimetric (RaXPol) Doppler radar system. J. Atmos. Oceanic Technol., 30, 1398-1413, https://doi.org/10.1175/JTECH-D-12-00166.1.

Potvin, C. K., A. Shapiro, T.-Y. Yu, J. Gao, and M. Xue, 2009: Using a low-order model to detect and characterize tornadoes in multiple-Doppler radar data. Mon. Wea. Rev., 137, 12301249, https://doi.org/10.1175/2008MWR2446.1.

Rasmussen, E. N., J. M. Straka, R. Davies-Jones, C. A. Doswell III, F. H. Carr, M. D. Eilts, and D. R. MacGorman, 1994: Verification of the Origins of Rotation in Tornadoes Experiment: VORTEX. Bull. Amer. Meteor. Soc., 75, 995-1006, https://doi.org/10.1175/ 1520-0477(1994)075<0995:VOTOOR > 2.0.CO;2.

Reynolds, G. W., 1971: Complication in estimating the magnitudes of tornado forces from damage analysis. Preprints, Seventh Conf. on Severe Local Storms, Kansas City, MO, Amer. Meteor. Soc., 179-182.

Rotunno, R., 2013: The fluid dynamics of tornadoes. Annu. Rev. Fluid Mech., 45, 59-84, https://doi.org/10.1146/annurev-fluid-011212-140639.

_ , G. H. Bryan, D. S. Nolan, and N. A. Dahl, 2016: Axisymmetric tornado simulations at high Reynolds number.J. Atmos. Sci., 73, 3843-3854, https://doi.org/10.1175/JAS-D-16-0038.1.

Schaefer, J. T., and J. G. Galway, 1982: Population biases in the tornado climatology. Preprints, 12th Conf. on Severe Local Storms, San Antonio, TX, Amer. Meteor. Soc., 51-54.

Shapiro, A., S. Rahimi, C. K. Potvin, and L. Orf, 2015: On the use of advection correction in trajectory calculations. J. Atmos. Sci., 72, 4261-4280, https://doi.org/10.1175/JAS-D-15-0095.1.

Simmons, K. M., and D. Sutter, 2005: WSR-88D radar, tornado warnings, and tornado casualties. Wea. Forecasting, 20, 301310, https://doi.org/10.1175/WAF857.1.

Snyder, J. C., and H. B. Bluestein, 2014: Some considerations for the use of high-resolution mobile radar data in tornado intensity determination. Wea. Forecasting, 29, 799-827, https:// doi.org/10.1175/WAF-D-14-00026.1.

, J. L. Salazer-Cerreno, H. B. Bluestein, N. A. Aboserwal, R. D. Palmer, and T. Y. Yu, 2015: Examining the effects of the ground on the radiation pattern of a parabolic reflector at very low elevation angles. 37th Conf. on Radar Meteorology, Norman, OK, Amer. Meteor. Soc., 166, https://ams.confex.com/ ams/37RADAR/webprogram/Paper276116.html.
Taylor, G. I., 1938: The spectrum of turbulence. Proc. Roy. Soc. London, 164A, 476-490.

Twisdale, L. A., 1982: Regional tornado data base and error analysis. Preprints, 12th Conf. on Severe Local Storms, San Antonio, TX, Amer. Meteor. Soc., 45-50.

Wakimoto, R. M., N. T. Atkins, K. M. Butler, H. B. Bluestein, K. Thiem, J. C. Snyder, and J. Houser, 2015: Photogrammetric analysis of the 2013 El Reno tornado combined with mobile X-band polarimetric radar data. Mon. Wea. Rev., 143, 2657-2683, https://doi.org/10.1175/MWR-D-15-0034.1.

_ - and Coauthors, 2016: Aerial damage survey of the $2013 \mathrm{El}$ Reno tornado combined with mobile radar data. Mon. Wea. Rev., 144, 1749-1776, https://doi.org/10.1175/MWR-D-15-0367.1.

Wind Science and Engineering Center, 2006: A recommendation for an enhanced Fujita scale. Texas Tech University, National Wind Institute, 111 pp., http://www.depts.ttu.edu/weweb/ efscale.pdf.

Wood, V. T., and R. A. Brown, 1992: Effects of radar proximity on single-Doppler velocity signatures of axisymmetric rotation and divergence. Mon. Wea. Rev., 120, 2798-2807, https://doi.org/ 10.1175/1520-0493(1992)120<2798:EORPOS >2.0.CO;2.

_ , and _ 1997: Effects of radar sampling on single-Doppler velocity signatures of mesocyclones and tornadoes. Wea. Forecasting, 12, 928-938, https://doi.org/10.1175/1520-0434(1997) $012<0928$ :EORSOS $>2.0$.CO;2.

Wurman, J., and M. Randall, 2001: An inexpensive, mobile, rapidscan radar. Preprints, 30th Int. Conf. on Radar Meteorology, Munich, Germany, Amer. Meteor. Soc., 98-100.

— C. Alexander, P. Robinson, and Y. Richardson, 2007: Lowlevel winds in tornadoes and potential catastrophic tornado impacts in urban areas. Bull. Amer. Meteor. Soc., 88, 31-46, https://doi.org/10.1175/BAMS-88-1-31.

_- K. Kosiba, P. Markowski, Y. Richardson, D. Dowell, and P. Robinson, 2010: Finescale single- and dual-Doppler analysis of tornado intensification, maintenance, and dissipation in the Orlenas, Nebraska, supercell. Mon. Wea. Rev., 138, 44394455, https://doi.org/10.1175/2010MWR3330.1.

- _ — , and P. Robinson, 2013: In situ, Doppler radar, and video observations of the interior structure of a tornado and the wind-damage relationship. Bull. Amer. Meteor. Soc., 94, 835-846, https://doi.org/10.1175/BAMS-D-12-00114.1.

,,--- , and T. Marshall, 2014: The role of multiple vortex tornado structure in causing storm researcher fatalities. Bull. Amer. Meteor. Soc., 95, 31-45, https://doi.org/ 10.1175/BAMS-D-13-00221.1. 